\title{
Chapter
}

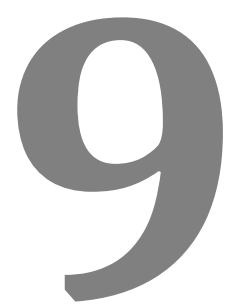

\section{NEAR INFRARED SPECTROSCOPY IN DRUG DISCOVERY AND DEVELOPMENT PROCESSES}

\author{
Yves Roggo, Klara Dégardin and Michel Ulmschneider
}

\section{Contents}

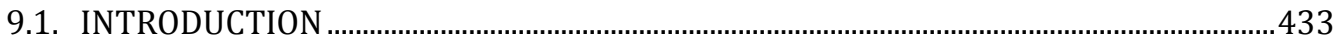

9.2. NIRS AND IMAGING: THEORY AND INSTRUMENTATION .................................................434

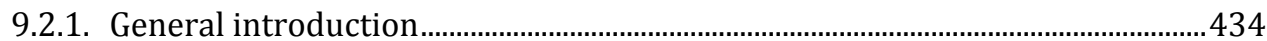

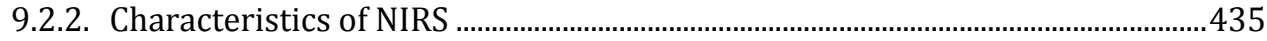

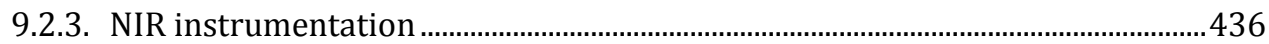

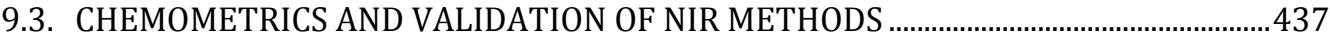

9.3.1. Development and validation of NIR methods ....................................................... 437

9.3.2. Mathematical preprocessing of spectroscopic data................................................441

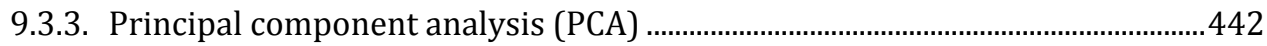

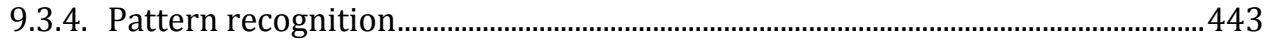

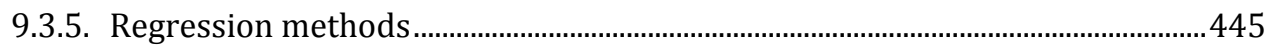




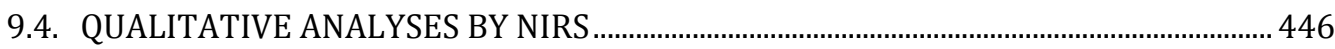

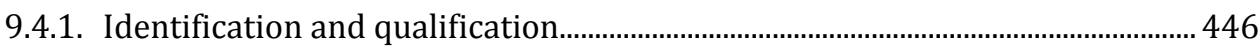

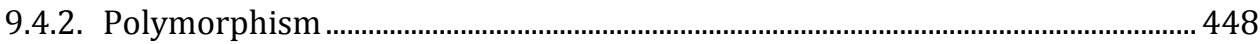

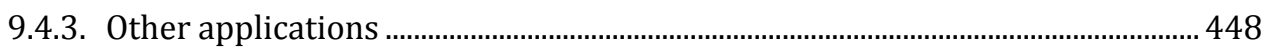

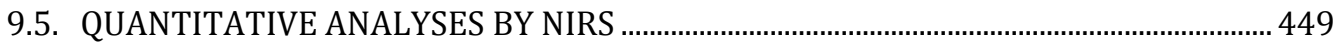

9.5.1. Physical parameters ....................................................................................................... 449

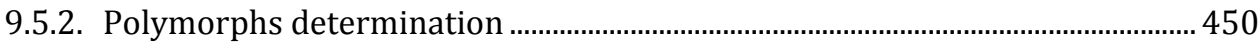

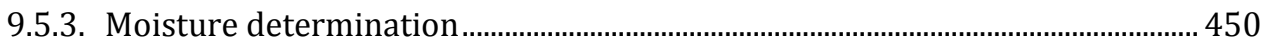

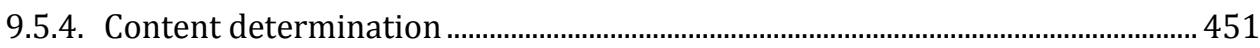

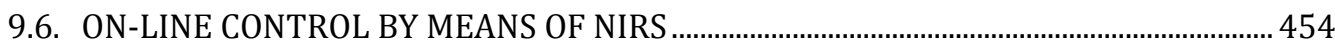

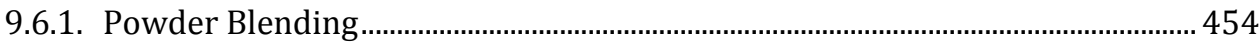

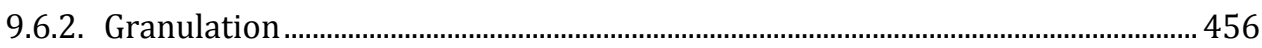

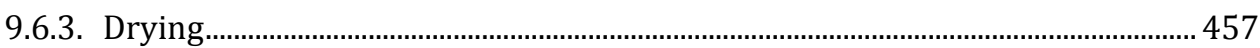

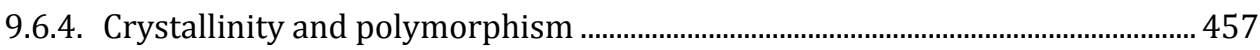

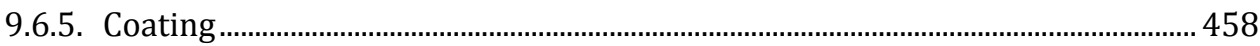

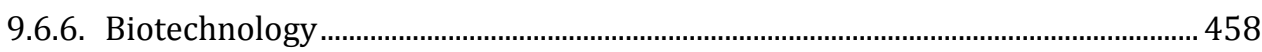

9.7. APPLICATIONS OF NIR SPECTRAL IMAGING................................................................... 460

9.7.1. General use of NIR chemical imaging for pharmaceutical applications ........... 460

9.7.2. Tablet composition analysis with NIR imaging ......................................................... 460

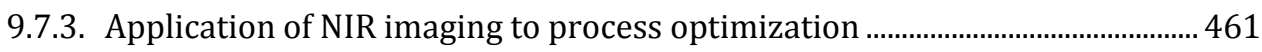

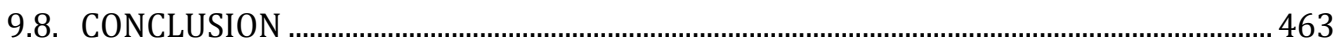

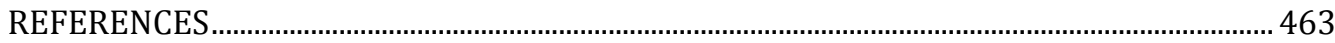




\subsection{INTRODUCTION}

Process analytical technology (PAT) has existed for many years in various industries. It was one of the objectives contained in the Pharmaceutical cGMPs for the 21st Century initiative launched in 2002 by the Food and Drug Administration (FDA). The FDA guideline defined PAT as a system for designing, analyzing and controlling pharmaceutical manufacturing through the measurement of critical quality and performance parameters. The measurements performed on raw and in-process materials or process parameters were intended to enhance final product quality. Both the FDA and the industry anticipated benefits over conventional manufacturing practices: higher final product quality, increased production efficiency, decreased operating costs, better process capacity, and fewer rejects [1].

Building quality into a pharmaceutical product has to be considered from the moment of a product's conception. PAT provides a motivating framework. If product quality requirements are understood and implemented from the outset, process failure after scale-up to commercial manufacturing will be much less likely. PAT greatly enhances process understanding. It continuously improves product quality, extends the acquired knowledge base for new projects, and shortens time to market. Trends in PAT were reviewed in 2011 [2], while Herdling and Lochmann have described and discussed PAT implementation in solid dosage form production [3]. PAT draws on the relevant basic sciences and a complex multiplicity of engineering and control technologies.

Near infrared spectroscopy (NIRS) is an important tool for PAT implementation [4], as it is increasingly used in pharmaceutical research and development.

In 1800, William Herschel discovered radiation beyond visible red light. However, it was many years before the NIR region was used for spectroscopy [5]. A wide overlap was observed in its bands, making them difficult to interpret. Karl Norris, an engineer at the U.S. Department of Agriculture, demonstrated the potential value of the NIR region for quantitative work by making measurements of agricultural products in the 1960s. The basic idea was to provide various research and production facilities with online NIR measurements of agricultural products, which was the field of interest at the time [5,6]. NIRS and chemometrics then spread to other domains, such as food [7], and the chemical [8] and oil industries [9], proving effective for both qualitative and quantitative analyses. 
NIRS is widely used in the pharmaceutical industry, with multiple practical applications and a massively increased presence in specialized journals.

NIRS is generally chosen for its speed, low cost, and non-destructiveness. It is a selective technique that is sensitive to the physical chemistry of the samples it analyzes. These can be measured intact or through packaging such as glass or plastic bags. NIR imaging is derived from NIRS and involves the acquisition of spatially located spectra that display compound distribution in the sample for analysis along with features such as moisture or particle size. Several reviews of NIRS are now available [10-13], together with its application to the development of solid dosage forms [14]. Interest in NIR has increased because of improved instrumentation and the development of fibre optics allowing non-contact measurement; it has also been boosted by the progress in computer science and data processing that has facilitated interpretation of NIR spectra, notably involving chemometrics, the body of mathematical and statistical techniques developed for the processing of chemical analytic data. Mid-IR (MIR) spectra, on the other hand, especially the absorbance bands, are directly readable thanks to chemical peak specificity.

The aim of this chapter is to present the theory and instrumentation of NIRS and NIR imaging, briefly describe some of the chemometric tools used in calibrating and validating NIR methods, and finally to focus on some NIR applications in the development process and drug discovery.

\subsection{NIRS AND IMAGING: THEORY AND INSTRUMENTATION}

\subsubsection{General introduction}

Infrared (IR) spectroscopy is a versatile tool applied to the qualitative and quantitative determination of molecular compounds of all types. The IR domain is subdivided into NIR, MIR, and far IR (FIR) with the following range limits (Figure 9.1):

Near IR: $\quad 780 \mathrm{~nm}$ to $2500 \mu \mathrm{m}\left(12800\right.$ to $\left.4000 \mathrm{~cm}^{-1}\right)$

Mid IR: $\quad 2500 \mu \mathrm{m}$ to $50 \mu \mathrm{m}\left(4000\right.$ to $\left.200 \mathrm{~cm}^{-1}\right)$

Far IR: $\quad 50 \mu \mathrm{m}$ to $500 \mu \mathrm{m}\left(200\right.$ to $\left.20 \mathrm{~cm}^{-1}\right)$

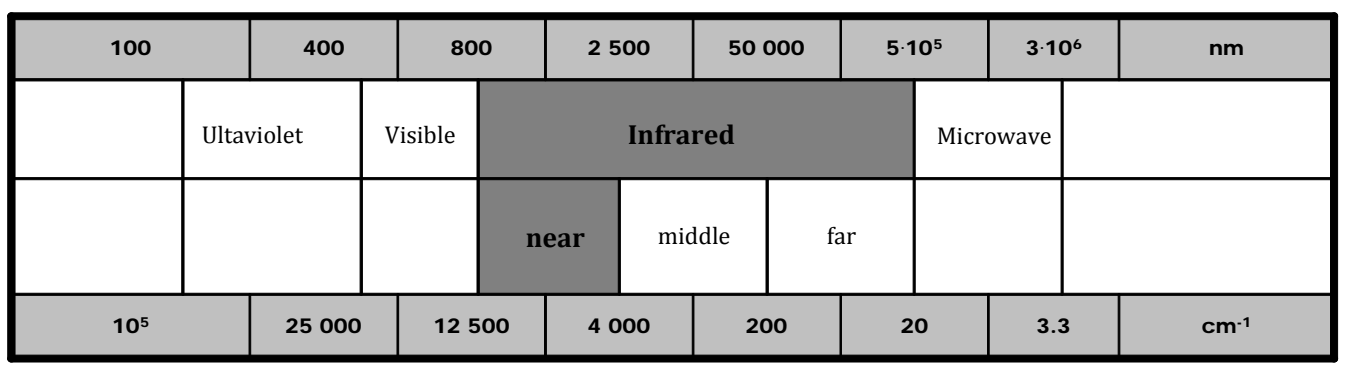

Figure 9.1: Electromagnetic spectrum - the infrared range 
MIR spectroscopy is by far the most widely used, with absorption, reflection, and emission spectra employed for both qualitative and quantitative analyses. FIR is primarily used for absorption measurements of inorganic and metal-organic samples.

The NIR region is particularly used for routine quantitative determination in complex samples containing functional groups of hydrogen bonded to carbon, nitrogen, or oxygen. This is of interest in agriculture, feed, food, and, more recently, pharmaceutical industries. NIRS was first used for the rapid screening of feed and food products for protein, moisture, starch, oil, lipid, and cellulose content.

\subsubsection{Characteristics of NIRS}

The main vibrations observed in the NIR region are those of $-\mathrm{CH},-\mathrm{OH},-\mathrm{SH}$, and -NH bonds. All the absorption bands result from overtones or combinations of the fundamental MIR bands [15,16]. The overtone and combination molecular absorptions found within the NIR region are much less intense than the fundamental IR absorptions.

Once the appropriate chemometric tools were developed, the NIR region turned out to be of great interest for industrial applications. NIRS is fast: once a method has been developed and validated, measurement only takes seconds. Samples require no preparation and can be measured as such, intact. They are available for further analysis since NIRS is non-destructive. Measurements can be performed on- and at-line. NIR spectrometers tend to be very robust. Glass fibre optics can be used to perform remote analysis by bringing radiation directly to the sample. The fibre optic probe can be in contact with the sample or immersed in it. As NIR measurements can be done through glass, this material can be used for windows, lenses, and any other optical components, which simplifies sampling.

Table 9.1 compares some pros and cons of NIR, IR and Raman spectroscopy.

Table 9.1. Comparison of near infrared (NIR), infrared (IR) and Raman spectroscopy

\begin{tabular}{cccc}
\hline Spectroscopy & NR & IR & Raman \\
\hline Signal range, $\mathrm{cm}^{-1}$ & $12000-4000$ & $4000-400$ & $4000-50$ \\
\hline Signal intensity & ++ & +++ & + \\
\hline Microscopic analysis & No & Yes & Yes \\
\hline Fiber optic interfacing & Yes & Yes (limit length) & Yes \\
\hline Sampling through glass & Yes & 0 & Yes \\
\hline Instrument robustness & +++ & + & ++ \\
\hline Chemical interpretation & With chemometric tools & Direct & Direct \\
\hline
\end{tabular}


NIRS has many more practical advantages in the pharmaceutical process thanks to numerous applications enabled by developments in instrumentation and sampling.

\subsubsection{NIR instrumentation}

The instrument design first depends on how measurements are performed. Diffuse reflectance and transmittance measurements are both used, although diffuse reflectance much more widely because of its ease of use. In the diffuse reflectance mode, radiation penetrates the particle surface layer, excites the vibrational modes of the analyte molecule, and is then scattered in all directions. Reflectance measurements penetrate only $1 \mathrm{~mm}$ to $4 \mathrm{~mm}$ into the solid sample surface. In this case the ordinate is the logarithm of the reciprocal of reflectance $R, \log (1 / R)$, where $R$ is the ratio of the intensity of radiation reflected from the sample to reflectance from a standard reflector. In transmittance mode, the entire path through the sample is integrated into the spectral measurement, thereby reducing error due to sample non-homogeneity. Transmittance is suitable for measuring through compact samples, like intact tablets, but surface scattering induces a loss of transmitted energy with the net effect being a decrease in the signal-to-noise ratio.

Many spectrometers have been specifically designed for the NIR range. The ideal instrument has both transmittance and reflectance capabilities. However, the choice is wide, especially when compared with MIR spectrometers. Grating, diode array, and Fourier transform (FT) instruments are the most sophisticated. FT spectrometers are mostly based on the Michelson interferometer, although other types of optical systems are also encountered. Tungsten-halogen lamps with quartz windows are used as sources while detectors are usually made of lead sulfide $(\mathrm{PbS}$ ) or arsine-gallium (AsGa). Handheld NIR spectrometers have also been developed that allow measurement in the field rather than in the lab [1719]. FT spectrophotometers are preferred for many applications because of their speed, reliability, and convenience. They appear to have better signal-to-noise ratios and a much larger energy throughput than dispersive instruments. Interferometric instruments also feature high resolutions, high accuracy, and reproducible frequency determination. Other designs include diode array detectors and NIR emitting diode sources. Acousto-optic tunable filters (AOTF) capable of microsecond scanning speeds are devices based on diffraction. Other techniques are available, such as ultrafast-spinning interference filter wheels, interferometers with no moving parts, and tunable laser sources.

Specific instruments are used for chemical imaging. A complete spectrum is acquired for each pixel, meaning that a hyperspectral image is in fact a data cube, i.e. a 3D matrix (Figure 9.2). Chemical imaging experiments yield an $X \times Y \times \lambda$ matrix or data cube, where $X$ and $Y$ are the spatial dimensions and $\lambda$ the spectral dimension [20]. In principle, it is possible to collect hyperspectral images with single-point detectors, i.e. classical mapping with microscopes. However, array 
detectors measure all pixels simultaneously, reduce recording time, provide uniform background, and improve the signal to noise ratio [21]. Figure 9.3 presents an example of a NIR image analyzer.

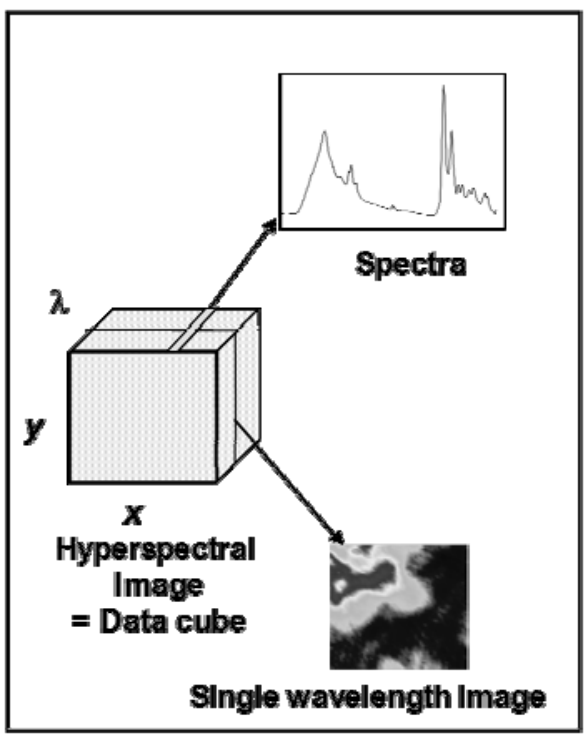

Figure 9.2. Near infrared (NIR) imaging concept [1]

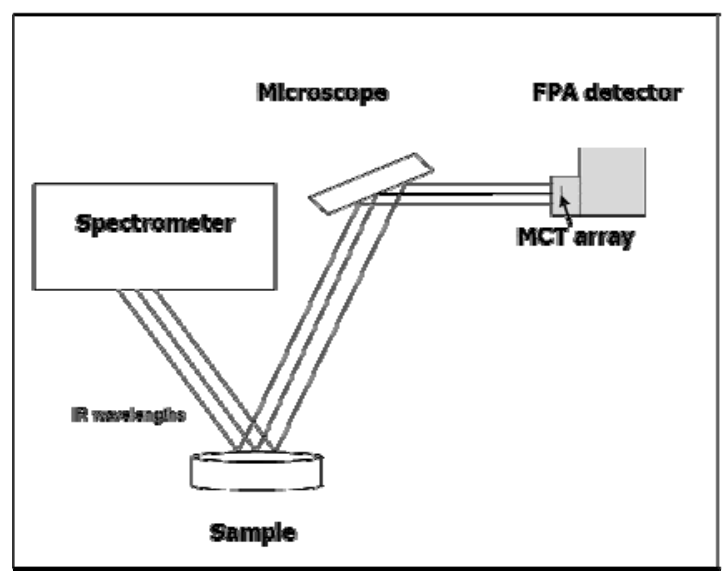

Figure 9.3. Near infrared (NIR) imaging [1]

\subsection{CHEMOMETRICS AND VALIDATION OF NIR METHODS}

Chemometrics [22-24] is used to select the optimal experimental procedure and processing technique for chemical analytic data. It draws on a variety of specialties, including experimental design, data extraction (modeling, regression, classification, hypothesis testing), and techniques for understanding chemical mechanisms (see review by Lavine [25] and many textbooks [26-28]).

\subsubsection{Development and validation of NIR methods}

\section{Sample selection}

A NIR model is built and validated with several sample sets, beginning with the calibration set used to compute the model. The second validation set is used to evaluate the model's ability to predict unknown samples. Both sets must be independent, i.e. they must consist of samples from different batches. Set selection and preparation are critical issues. For instance, for quantitative methods the analyst must collect or prepare samples which span the complete range of constituent concentrations spread out as evenly as possible. Calibration sets must comprise a correctly distributed number of sample types. Spectro- 
scopic measurements with calibration samples should be performed under conditions approximating to these for routine samples.

Table 9.2 offers an example of a quantitative NIR validation plan. Qualitative methods require testing only for specificity and robustness.

Table 9.2. Tests for quantitative near infrared (NIR) validation parameters

\begin{tabular}{|c|c|}
\hline $\begin{array}{l}\text { Parameters } \\
\text { for validation }\end{array}$ & Tests \\
\hline \multirow{2}{*}{ Specificity } & Predict all samples of the external validation set using the calibrated model \\
\hline & Predict other samples (challenge samples) \\
\hline Linearity & $\begin{array}{l}\text { Plot and determine slope and intercept of NIR predicted values }(y) \text { versus } \\
\text { reference values }(x) \text { of calibration and validation sets }\end{array}$ \\
\hline \multirow{4}{*}{ Accuracy } & Determine the standard error of prediction (SEP) with validation set \\
\hline & Compare SEP and accuracy of the reference method. \\
\hline & $\begin{array}{c}\text { Compare reference and calibration set NIR results using paired sample t-tests } \\
\text { to test for significant differences }\end{array}$ \\
\hline & Recovery experiments \\
\hline \multirow[b]{2}{*}{ Precision } & Repeatability: e.g. 10 times on the same day \\
\hline & $\begin{array}{c}\text { Intermediate precision: } 3 \text { different analysts assaying the same production } \\
\text { sample on } 3 \text { separate days }\end{array}$ \\
\hline Robustness & $\begin{array}{l}\text { Test errors associated with sampling speed, temperature, sample position, } \\
\text { fibre optic parameters, etc. }\end{array}$ \\
\hline
\end{tabular}

Once the model has been constructed and validated it can be routinely used. It can be run on the device used during development or on another device; in which case transferability must be ensured (some adjustment is usually necessary).

\section{Use of regression for NIR quantitative methods}

Before a quantitative model is computed, the purpose of the calibration and its minimal accuracy and limits of validity need to be established. A robust model presupposes the design of a range of samples comprising adequate lateral variation [29-31].

Calibration is the fitting stage: the main data set, containing only the calibration samples, is used to compute model parameters such as principal components (PCs) and regression coefficients. The models must be validated to get an idea of how well a regression model performs if used to predict new, unknown samples. A validation set consisting of samples with known variable values is used. The most common distribution allocates two-thirds of the samples to the calibration set and one third to the internal validation set. Sample selection studies have compared Kennard-Stone and successive projections algorithms, random sampling, and full cross-validation for use with multiple linear regression (MLR) and partial least squares (PLS) models [32,33].

During the validation step, only the spectral information is introduced into the model, from which response values are predicted and compared to the known 
values of the reference method. If the uncertainty of prediction is reasonably low, the model can be considered usable. Independent test-set validation and crossvalidation are the most current methods of estimating prediction error. With cross-validation, the same samples are used for both model estimation and testing. This represents an alternative form of validation if sample numbers are small. The method consists in leaving out a few samples from the calibration set and calibrating the model on the remaining data. The left-out sample values are then predicted and the corresponding prediction residuals computed. The process is repeated with another calibration subset, and so on until every object has been left out. Figure 9.4 illustrates the steps required for complete modeling.

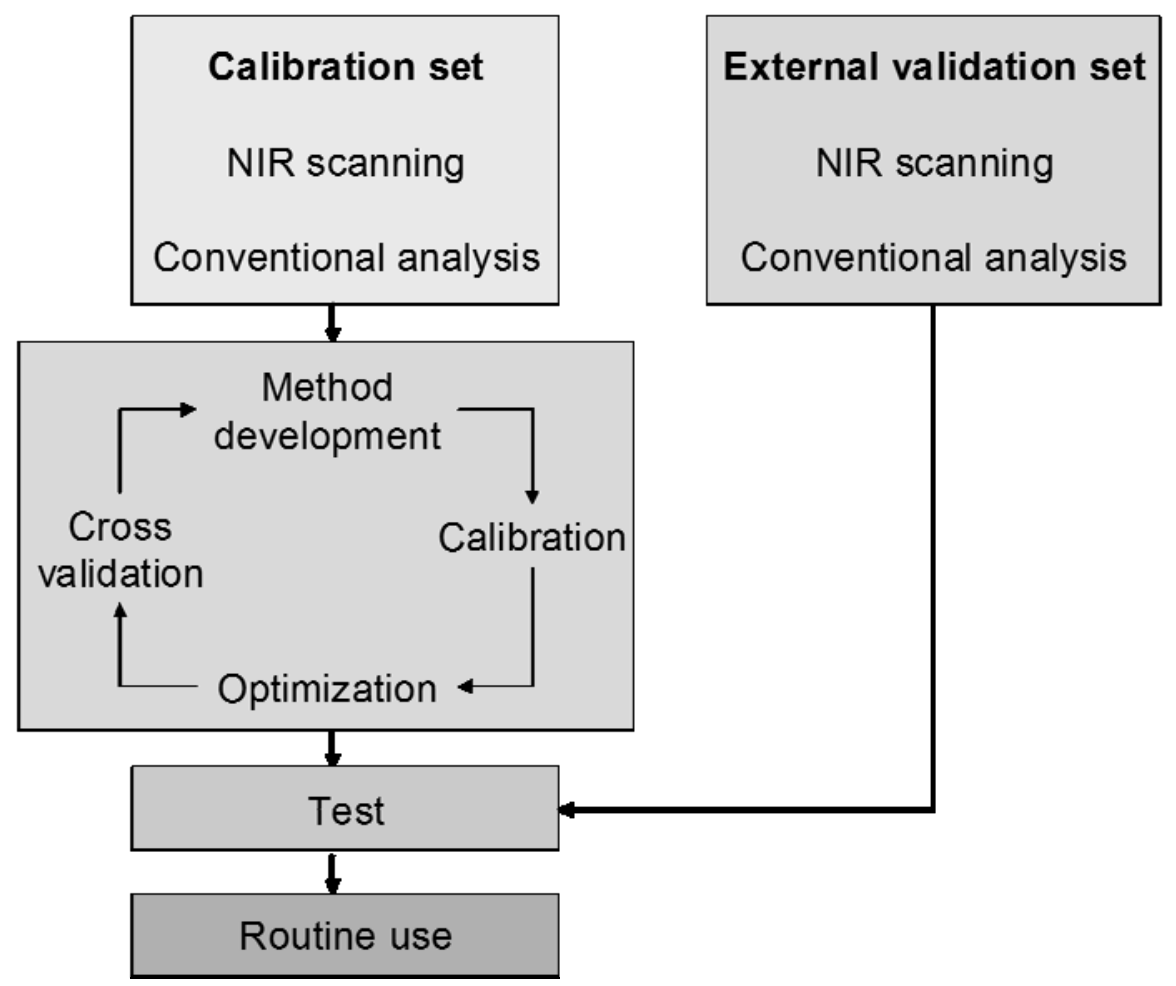

Figure 9.4. Principle of multivariate calibration (NIR - Near infrared)

The predicted $Y$ values are then compared with the observed $Y$ values (Figure 9.5). This generates a prediction residual that can be used to compute a validation residual variance, or a measure of the uncertainty of future predictions.

Goodness of fit of a prediction model can be evaluated by further criteria such as the low standard error of calibration (SEC), low SEP, high correlation coefficient $\left(R^{2}\right)$, and low bias. SEC, standard error of cross-validation (SECV), SEP, bias, slope, and SEP with bias correction (SEP(C)) are the criteria of model accuracy (formu- 
las in Figure 9.6). Naes and co-workers have described the statistical strategies available [34] and there are other examples of NIR validation methods [35].

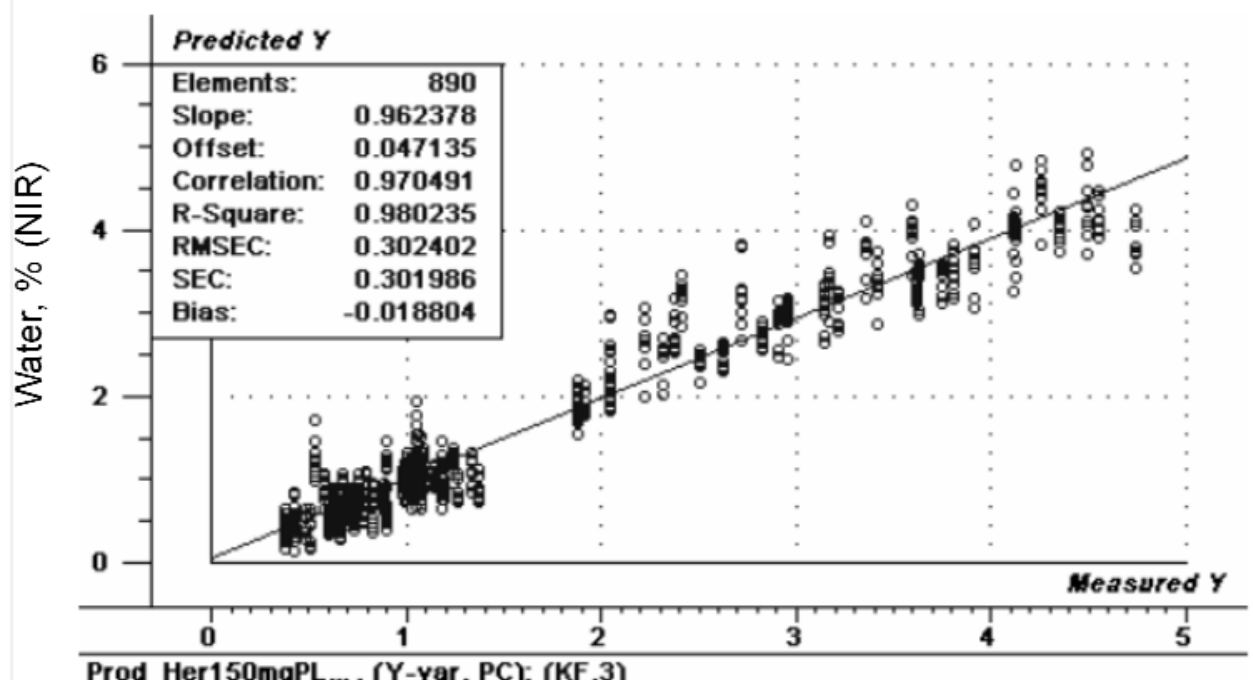

Prod_Her150mgPL... , (Y-var, PC): (KF,3)

Water, \% (KF)

Figure 9.5. Example of a regression performed on near infrared (NIR) spectra and reference results (KF - Karl Fischer titration)

$?_{y}=\sqrt{\frac{\sum_{j=1}^{m}\left(y_{j}-\bar{y}\right)^{2}}{m-1}}$

$\operatorname{cov}(y, y)=\sqrt{\frac{\sum_{j=1}^{m}\left(y_{j}-\bar{y}\right)\left(y_{j}^{\prime}-y^{\prime}\right)}{m-1}}$

$\mathrm{R}=\frac{\operatorname{cov}\left(\mathrm{y}^{\prime}, \mathrm{y}\right)}{\sigma_{\mathrm{y}^{\prime}} \cdot \sigma_{\mathrm{y}}}$

$S E C=\sqrt{\frac{\sum_{j=1}^{m}\left(y_{j}-y_{i}^{\prime}\right)^{2}}{m-1-q}}$

$S E P=\sqrt{\frac{\sum_{j=1}^{n}\left(y_{j}-y_{j}^{\prime}\right)^{2}}{n}}$

Bias $=\frac{\sum_{j=1}^{n}\left(y_{j}-y_{j}^{\prime}\right)}{n}$

$\begin{array}{ll}\text { where } & =\text { reference value for the sample } \# \mathrm{j} \\ \mathrm{y}_{\mathrm{j}} & =\text { predicted value for the sample } \# \mathrm{j} \\ \mathrm{y}_{\mathrm{j}}^{\prime} & =\text { number of samples in calibration } \\ \mathrm{m} & =\text { number of samples in validation } \\ \mathrm{n} & =\mathrm{PC} \text { number } \\ \mathrm{q} & =\text { standard deviation }\end{array}$

Figure 9.6. Formulas for goodness of fit criteria (SEC - standard error of calibration; SEP - standard error of prediction) 


\subsubsection{Mathematical preprocessing of spectroscopic data}

Spectral raw data may have a distribution or shape that is not optimal for analysis. Background effects, baseline shifts, or measurements under different conditions can complicate the extraction of information. It is thus important to minimize the noise introduced by such effects with preprocessing operations. These include centering, weighting, and numerous mathematical transformations.

Mean-centering consists of subtracting average spectra from each spectrum to ensure that all results will be interpretable in terms of variation around the mean. Weights based on the standard deviation (SDev, square root of the variance) may also be used for scaling. A possible weighting option is the $1 / \mathrm{SDev}$ standardization, which gives all variables the same variance so that they have the same chance to influence estimation of the components. This is advisable if the variables are measured with different units, have different ranges, or are of different types. It is also possible to fix a constant weight for each variable manually. This involves stretching and shrinking by measuring a position relative to the extremes. However, this emphasizes the relative influence of unreliable or noisy attributes. Smoothing is relevant for variables which themselves are a function of some underlying variable, for instance time. It is also one of the first operations performed on recorded NIR spectra. It removes as much noise as possible without degrading important information content. Polynomial smoothing, also called Savitzky-Golay smoothing, involves least square fitting of a polynomial equation to a window of $n$ sequentially selected spectral data points. Normalization is a family of transformations which are computed sample-wise, in this case to improve specific properties. Mean normalization is the classic algorithm. It consists in dividing each row of a data matrix by its average, thus neutralizing the influence of possible hidden factors. Maximum normalization is an alternative procedure which divides each row by its maximum absolute value instead of the average. Multiplicative scatter correction (MSC) is a transformation method used to compensate for additive and/or multiplicative effects in spectral data. It successfully treats multiplicative scattering and similar effects such as path length problems, offset shifts, and interference. Derivation is typically relevant for spectral data that are a function of some underlying variable influencing absorbance at various wavelengths. It is also a simple but powerful technique for magnifying fine structure in raw spectra lacking structure, which is common in NIRS. By increasing the order of derivation, band structure resolution is increased. The Savitzky-Golay algorithm permits computation to higher order derivatives, including a smoothing factor which determines how many adjacent variables will be used to estimate the polynomial approximation for derivation. Norris derivation is an alternative algorithm for computing first derivatives. A baseline correction algorithm is the standard normal variate (SNV) method which does not affect overall spectra layout. 


\subsubsection{Principal component analysis (PCA)}

Large tables contain information partly hidden by data complexity; a feature typical of NIR spectra collection. PCA is a projection method that visualizes all the information contained in a data table. It can be used to show in what respect one sample differs from another, which variables contribute the most to that difference, and whether these variables contribute in the same way, and are correlated or independent from each other. It also reveals sample patterns and outliers.

PCA modeling forms the basis for several classification and regression methods. The underlying idea is to replace a complex data set by a simpler version having fewer dimensions, but still fitting the original data closely enough to be considered a good approximation. Two samples can be described as similar if they have close values for most variables. From a geometric perspective, in the case of close coordinates in the multidimensional space of variables, the two points are located in the same area (Figure 9.7).
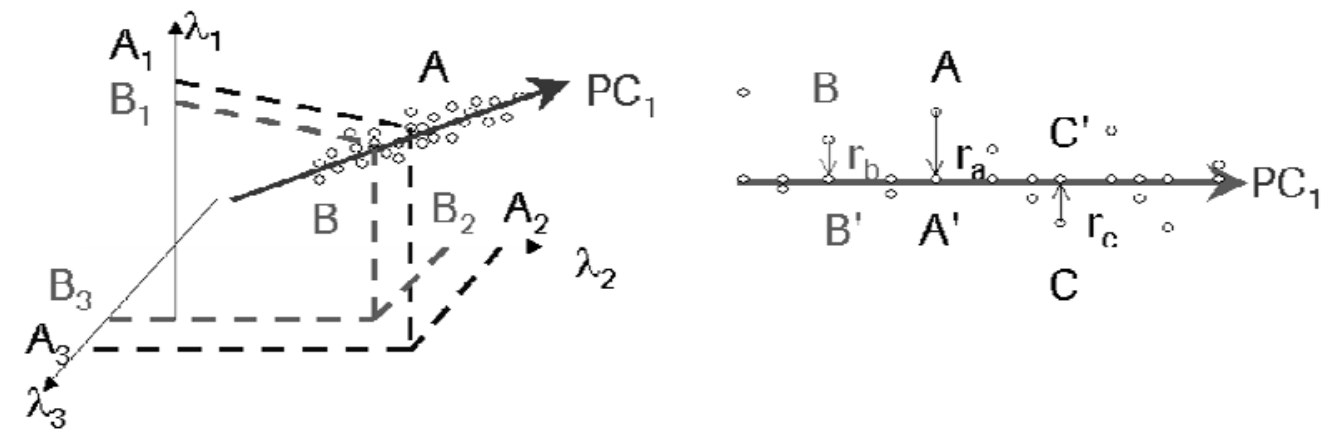

Figure 9.7. The geometric concept of principal component analysis (PCA)

A, B, C - three samples; $\lambda$ - wavelength; PC - principal component

PCA consists in finding the directions in space - known as principal components (PCs) - along which the data points are furthest apart. It requires a linear combination of the initial variables that contribute the most to making the samples different from each other. PCs are computed iteratively, with the first PC carrying the most information, i.e. the most explained variance, and the second PC carrying most of the residual information not taken into account by the previous PC, and so on. This process can go on until as many PCs have been computed as there are potential variables in the data table. At that point, all between-sample variation has been accounted for, and the PCs form a new set of axes. Usually, only the first PCs contain pertinent information, with the last PCs being more likely to describe noise. Deciding on the number of components to retain in a PCA model involves a compromise between simplicity, completeness, and effectiveness.

Each PCA component is characterized by attributes, e.g. variances, loadings, and scores. Variances are data scattering measure showing how much information 
the PCs take into account. Residual variance designates the variation in the data that remains to be explained once the current PC has been taken into account, while explained variance measures the variation in the data accounted for by the current PC. Loadings describe the data structure in terms of variable correlations. Variables with high loadings for a given PC contribute greatly to the meaning of that particular PC. Scores describe the data structure in terms of sample patterns and emphasize differences or similarities. Each sample has a score on each PC which is the coordinate of the sample on the PC. It describes the major features of the sample, relative to the variables with high loadings on the same PC. Samples with close scores along the same PC are considered as similar because they have close values for the corresponding variables.

Once NIR spectra have been measured, building and using a PCA model involves three steps: selecting the appropriate preprocessings, running the PCA algorithm and diagnosing the model, and interpreting the loading and score plots (Figure 9.8) [36].

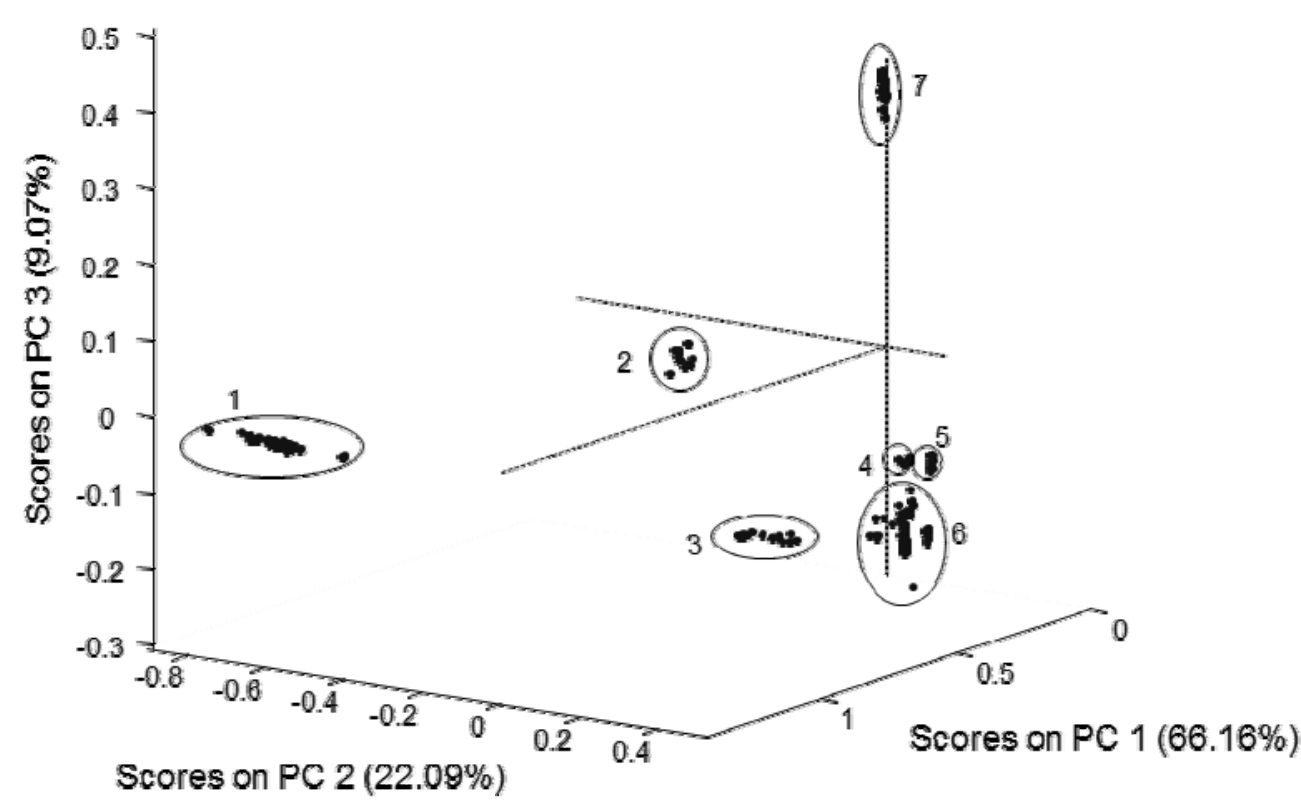

Figure 9.8. Principal component analysis (PCA) score plot identifying seven clusters with the first three principal components (PCs) [36]

\subsubsection{Pattern recognition}

Pattern recognition methods are often applied in chemistry [37], biology [38], and food science [39]. The main goal is to assign new samples reliably to preexisting classes. Classification results can also be used as a diagnostic tool to identify the most important variables or find outliers. Applications include predicting whether a pharmaceutical product meets specified quality 
requirements or more generally confirming substance identity. PCA and discriminant analysis are techniques that have found extensive use in NIR analysis for this purpose.

The classification techniques can be divided into two categories: unsupervised and supervised. In unsupervised classification, samples are classified without prior knowledge, except the spectra. The spectroscopist's job is to explain the clusters obtained. Many clustering algorithms can be used, such as the hierarchical method (Figure 9.9) [36].

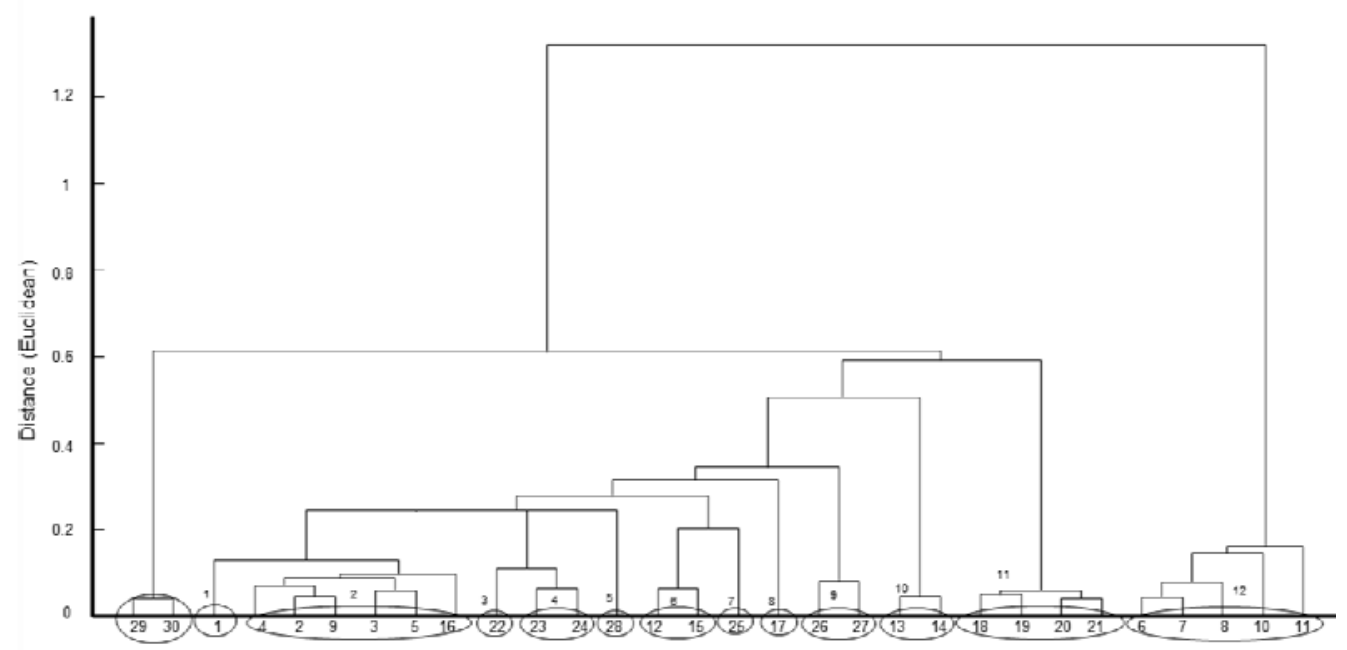

Figure 9.9. Hierarchical cluster analysis (HCA) dendrogram [36]

Supervised methods are those requiring prior knowledge, i.e. the category membership of samples. Thus, the classification model is developed on a training set of samples with known categories [28]. Then the model performance is evaluated by comparing the classification predictions to the true categories of the validation samples. Therefore, mathematical models are computed in a first step with a calibration set containing spectra and class information. Feature extraction methods such as PCA are often applied before cluster analysis.

Current methods for supervised pattern recognition are numerous. Typical linear methods are linear discriminant analysis (LDA) based on distance calculation, soft independent modeling of class analogy (SIMCA), which emphasizes similarities within a class, and PLS discriminant analysis (PLS-DA), which performs regression between spectra and class memberships. More advanced methods are based on non-linear techniques, such as neural networks or support vector machines (SVM). 


\subsubsection{Regression methods}

\section{From univariate to multivariate regression}

Regression concerns all methods attempting to fit a model to the observed data. In spectroscopy the simplest method of quantitative calibration is based on a single independent variable, e.g. wavelength, since a sample attribute such as analyte concentration is a linear function of absorbance at a given wavelength. This is called univariate regression. In this approach, a wavelength is selected when it shows a high degree of correlation between concentration and absorbance. Correlation is an indicator of how well the calibration describes the data set. The linear relationship permits direct and visual estimation of goodness of fit, thus enhancing the analyst's trust in their data collection. Where pharmaceutical samples are concerned, the linear approach rapidly reaches its limits and another approach, multivariate regression, is required.

Multivariate regression takes several predictive variables simultaneously into account for greater accuracy. The fitted model may thus be used to describe the relationship between two groups of variables, or to predict the values of unknown samples.

\section{Multiple linear regression (MLR)}

MLR extends linear regression to one wavelength by least squares, with more than one wavelength selected to perform a calibration. The method requires independent variables in order to explain the data set. More samples than predictors are necessary and no missing values must be present in the data table. If it complies with these conditions, MLR will approximate the response values by a linear combination of predictor values, yielding regression coefficients. It is worth mentioning that MLR is the only multivariate method for which formal statistical tests of significance for regression coefficients are available. Y variances provide the relevant measure of MLR model performance, showing how much variation remains in the observed response after the modeled part is removed, and acting as an overall measure of misfit.

\section{Principal component regression (PCR) and PLS regression}

With PLS multivariate regression, spectral and constituent data are modeled simultaneously according to an iterative algorithm. PCR and PLS are both projection methods, like PCA. PLS1 deals with only one response variable at a time (like MLR and PCR). PLS2 handles several responses simultaneously. The unexplained part of the data set is made up of residuals. The original data are combined in factors or PCs. A critical step in PLS modeling is the selection of the number of factors. Selecting too few factors will provide an inadequate explanation of variability, while too many factors will cause overfitting and instability in the resulting calibration. Coefficients, loadings and scores are calculated to indicate the extent of original data involvement in the computation of each factor. In a final step, the amount of variance modeled is maximized for 
each factor and the residuals are minimized. Either an additional and independent data set is used or the training data set is split into subsets for continuous internal validation at each iterative step (cross-validation). In addition, the resulting PLS calibration must prove able to predict unknown samples

\subsection{QUALITATIVE ANALYSES BY NIRS}

Qualitative analyses mean classifications of samples based on pattern recognition methods and their NIR spectra. Classification is simply a matter of finding out whether new samples are similar to classes of samples that have been used to make the model. If a new sample fits a particular model well, it is said to be a member of that class. Many analytical tasks fall into this category. The aim of this part is to present an overview of pharmaceutical applications for qualitative analyses, especially the identification and qualification of raw and final material.

\subsubsection{Identification and qualification}

Analysis of raw materials and pharmaceutical intermediates

The International Conference on Harmonisation of Technical Requirements for Registration of Pharmaceuticals for Human Use (ICH) guidelines describe the importance of identity tests [40-43]. Pharmacopoeias [44] have selected analytical methods like HPLC, optical rotation, and colorimetry to identify raw materials. The NIR application for qualitative analysis is, however, now also described by the European Pharmacopoeia in chapter 2.2.40. The qualification of a sample will determine whether it is within the normal variability range or subject to overlimit deviations. Key quality parameters can be evaluated for this purpose [45]. Distance based methods are often applied for the qualification of products. If the sample belongs to the same population as the reference product, then there is a probability of $99.7 \%$ that the distance will be less than three times the standard deviation. If the maximum distance is higher than that value, then the sample is from a different population.

The identification of incoming raw materials is now a common NIRS application [46-48] thanks to the limited sample preparation it requires (Figure 9.10). A lot of publications describe the application of NIRS for the control of excipients, active pharmaceutical ingredients (APIs) and final products. Ulmschneider and co-workers applied NIRS to identify different types of starch, sugar, cellulose, intermediates and APIs with PCA and cluster calibration [49-52]. NIRS was used by Ebube and co-workers to differentiate between Avicel products [53]. The discrimination of cellulose [54] is indeed statistically significant. Cellulose ethers were identified by NIRS, but the separation of methylcellulose and cellulose ethers with methyl or hydroxyalkyl groups was not possible. Several types of poly(vinylpyrrolidone) (povidones) are characterized by their viscosity measured in water. Kreft and co-workers [55] have developed a SIMCA method for their differentiation by NIRS. The identification of raw materials can be 
performed directly at-line in the dispensing or at the reception in the warehouse. NIRS is now used in the manufacture of solids and also in biotechonolgy production for identification of cell culture medium [56]. NIRS identification is used in manufacturing plants and during process development.

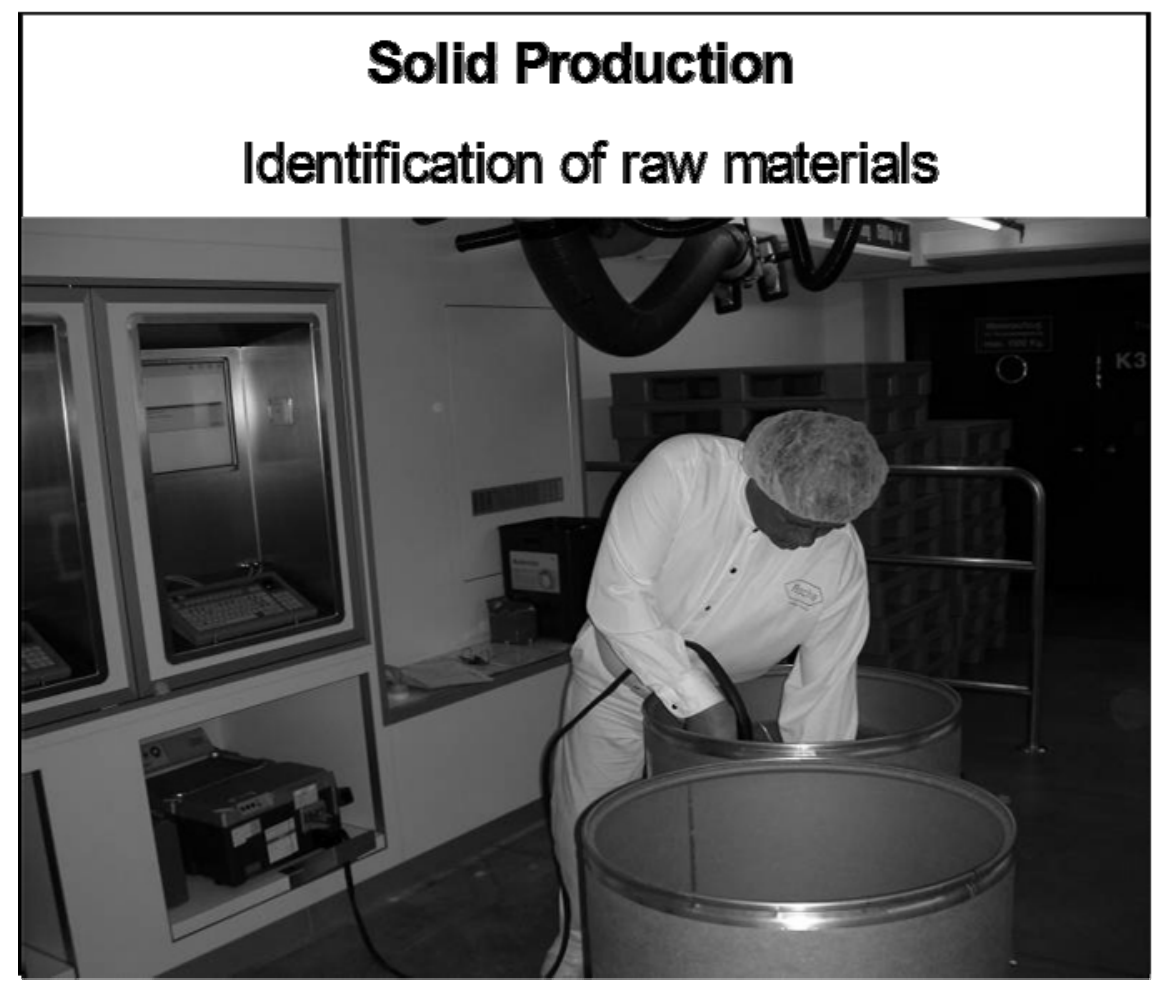

Figure 9.10. Raw material identification through plastic bags with a fibre optic probe in a warehouse

Final product: identification and counterfeit detection

Discriminating substances in tablet matrixes is possible and was studied by Chong and co-workers [57]. NIR can be and is commonly used for identity tests in quality control departments [58]. NIR transmission spectroscopy combined with chemometric methods is moreover applicable to confirm the identity of clinical trial tablets [59].

NIRS is besides a suitable method for the fast detection of counterfeit medicines [60-66]. In Figure 9.11 a discriminant analysis model is presented that was computed on NIR spectra of authentic and suspect capsules. For example, genuine capsules labelled as types 1 and 2 could be separated after pretreatment. Counterfeits of both types of capsules were successfully separated from the genuine capsules as a result of Mahalanobis distance. The same procedure can be applied to detect placebos in clinical studies. 


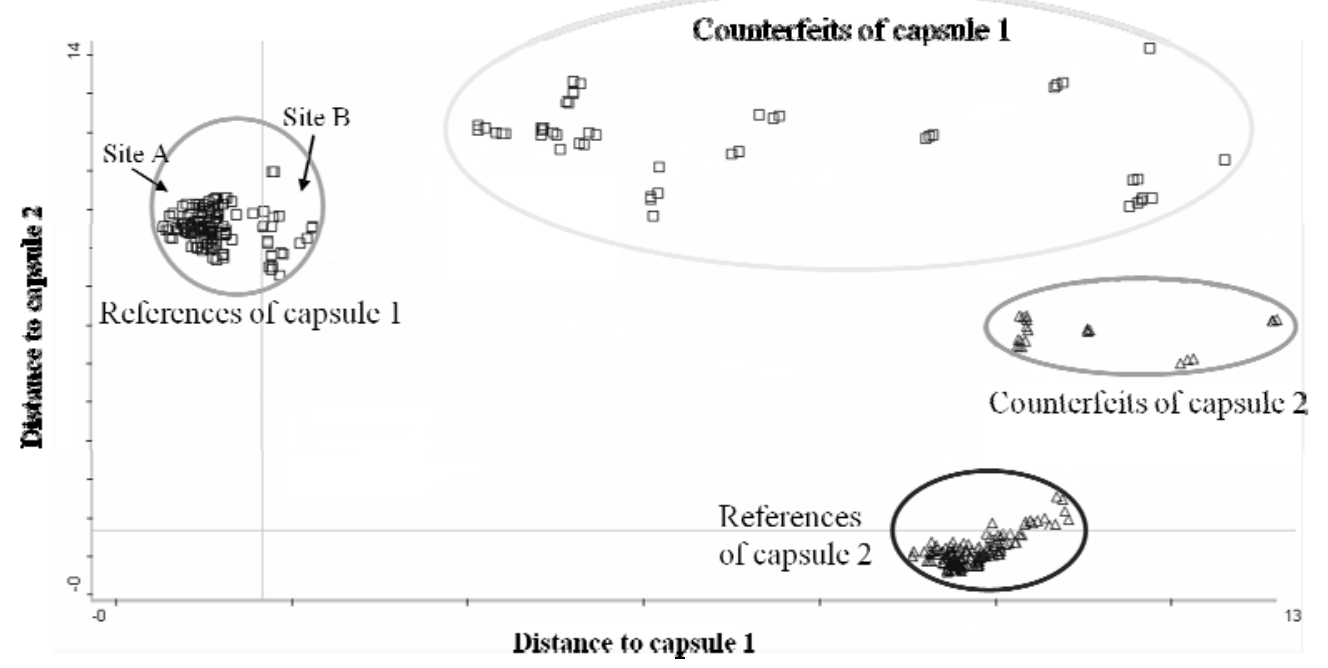

Figure 9.11. Discriminant Analysis performed on near infrared (NIR) spectra of genuine (reference) and counterfeit capsules

\subsubsection{Polymorphism}

The ability of a substance to be present in different crystalline forms is called polymorphism. The solid state properties have an influence on the stability and dissolution properties of the pharmaceutical product. This needs to be controlled by analytical methods, which could also help in galenical production and development. Raman spectroscopy has been successfully applied to characterize API polymorphic forms, but this pharmaceutical issue can be solved by NIRS as well. Information concerning the crystalline form of miokamycin could, e.g. be determined by NIRS [67]. This tool could moreover improve the understanding of physical forms of theophylline [68] and polymorphic transformation of pazopanib hydrochloride [69]. In addition, the characterization and analysis of azithromycin, an antibiotic derived from erythromycin A, was studied by Blanco and co-workers [70]. The suitability of NIRS to follow changes in both the amorphous and crystalline forms of lactose at room temperature was also investigated [71]. Also, their differentiation was possible by studying NIR frequencies of water peaks. A comprehensive review dealing with polymorph analysis was recently published [72].

\subsubsection{Other applications}

After the presentation of the main qualitative applications of NIRS, other studies can be mentioned as well, such as the discrimination of production sites of tablets. This is valuable to manufacturers, customers and industry regulations. Yoon and co-workers [73] used PCA for this purpose, which score plots showed that spectra of tablets originating from different manufacturing sites are statistically different. 
NIRS and chemometrics are besides combined to understand process (Figure 9.12) and dissolution issues [74]. This shows how NIRS can be useful for understanding batch differences due to variations in process conditions. Beginning with a qualitative analysis of the potential application of NIRS and IR imaging for solids analysis, the ability of NIRS to detect the effects of melt granulation time-temperature gradient, compaction force, coating formulation and coating time was tested on pilot production samples.

As a new field of application, NIR qualitative analyses of biotechnology products are also increasingly performed such as identification of bacteria strains $[75,76]$.

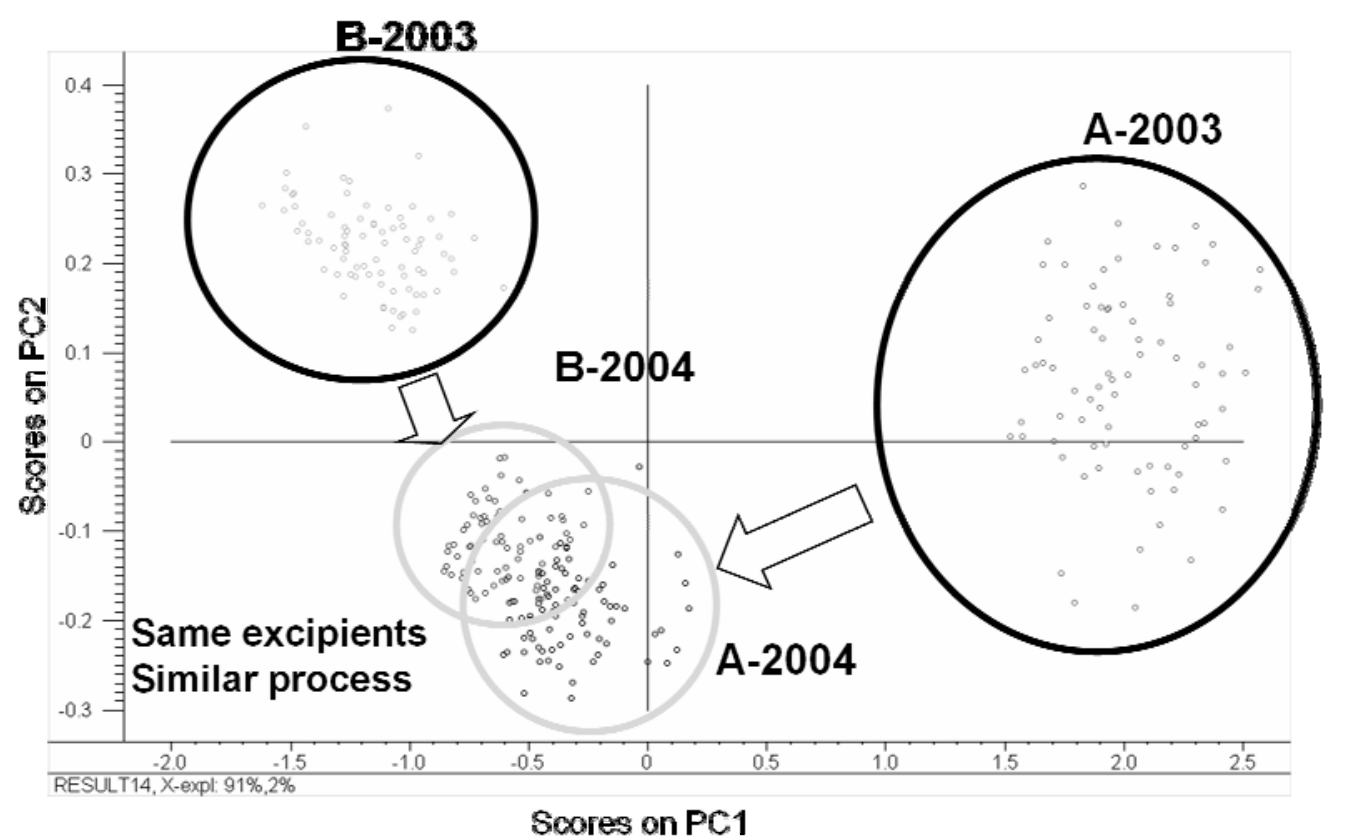

Figure 9.12. Comparison of production sites before and after process harmonization

\subsection{QUANTITATIVE ANALYSES BY NIRS}

Once the classification of samples has been achieved it can be useful to know more precisely to what extent they differ. Therefore, the development of a quantitative model appears useful. Historically, the first models were computed for the determination of sample moisture, according to two strong water bands absorbing at 1450 and $1940 \mathrm{~nm}$ [16].

\subsubsection{Physical parameters}

NIR spectra contain information about the chemical and physical properties of the analyzed samples. NIRS is nowadays used to determine a large panel of physical parameters on powders and tablets. Various biopharmaceutical parameters can be quantitatively analyzed by NIRS, such as hardness (for 
instance, tablet hardness [77]), particle size [78-80], compaction force, flow properties [81] and dissolution rate [82,83].

Tablet hardness is determined in different studies with PLS and MLR methods. Morisseau and co-workers [84] used these well established regression methods for this purpose and concluded that the accuracy of the results are highly dependent on the products and their formulation. In a study on drug content and tablet hardness by Chen and co-workers [85], good results were achieved on two different models computed by an artificial neural network (ANN). The correlation between compression force and NIR spectra at a specific wavelength is

demonstrated by Guo [86]. Blanco and co-workers [87] have more recently shown the possibility of predicting the pressure of compaction on a lab sample by using a PLS model.

The use of different regression methods enable chemists to follow the percentage of the drug released in the medium by a tablet. The dissolution profile was determined with NIRS by Donoso and Ghaly [88].

Berntsson published results on the determination of particle size when measuring powder blends with NIRS in reflectance [89,90]. In 2004, Otsuka analyzed the scattering effect due to particle size that was measured with a PCR model [91].

\subsubsection{Polymorphs determination}

The polymorphic form of a product is a key parameter as it influences its dissolution properties. The determination of the ratio between amorphous and crystalline forms of products is usually performed by X-Ray diffraction. Several studies showed that this analysis can also be done by NIRS [92-94]. Bai [95] observed great agreement between NIRS and X-Ray in the analysis of glycine crystallinity. The SEP obtained by NIRS was $3.2 \%$ and this tool was proven to detect crystallized glycine at a lower content than X-Ray diffraction. NIRS combined with regression methods has already been used for several polymorphism or crystallization applications [67,70,96-99].

\subsubsection{Moisture determination}

The quantitative analysis of moisture is one of the very first applications of NIRS in the pharmaceutical field. The existence of water in medicines is critical as it ensures stability. Water content is indicated by the presence of two important water bands at $1450 \mathrm{~nm}$ and especially around $1940 \mathrm{~nm}$ (Figure 9.13).

NIR spectroscopy is now used to determine the water content in powders or granules [100-102], tablets or capsules [103-105], as well as in lyophilised vials or solutions [106]. Use of NIRS in moisture determination is long established, therefore most of the relevant applications for this purpose are now implemented on-line (Figure 9.14). 


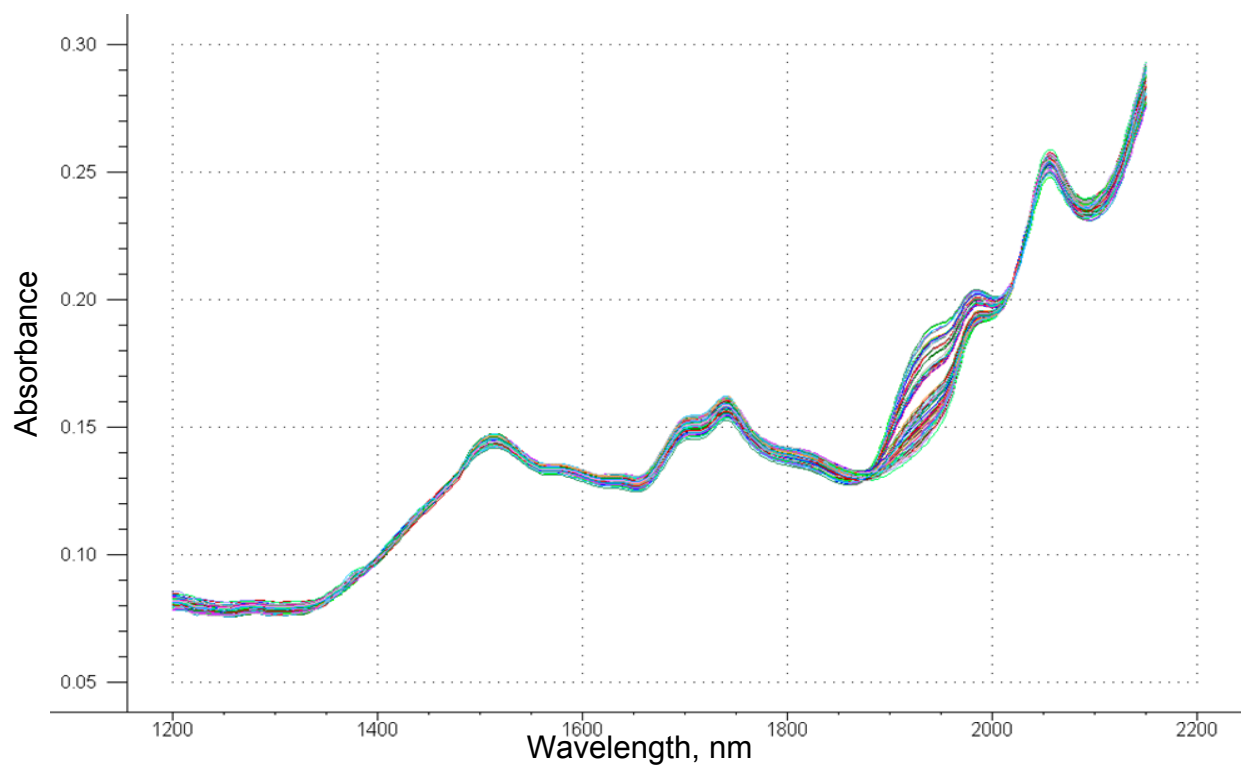

Figure 9.13. Water content evolution in lyophilised vials (peak at $1940 \mathrm{~nm}$ )
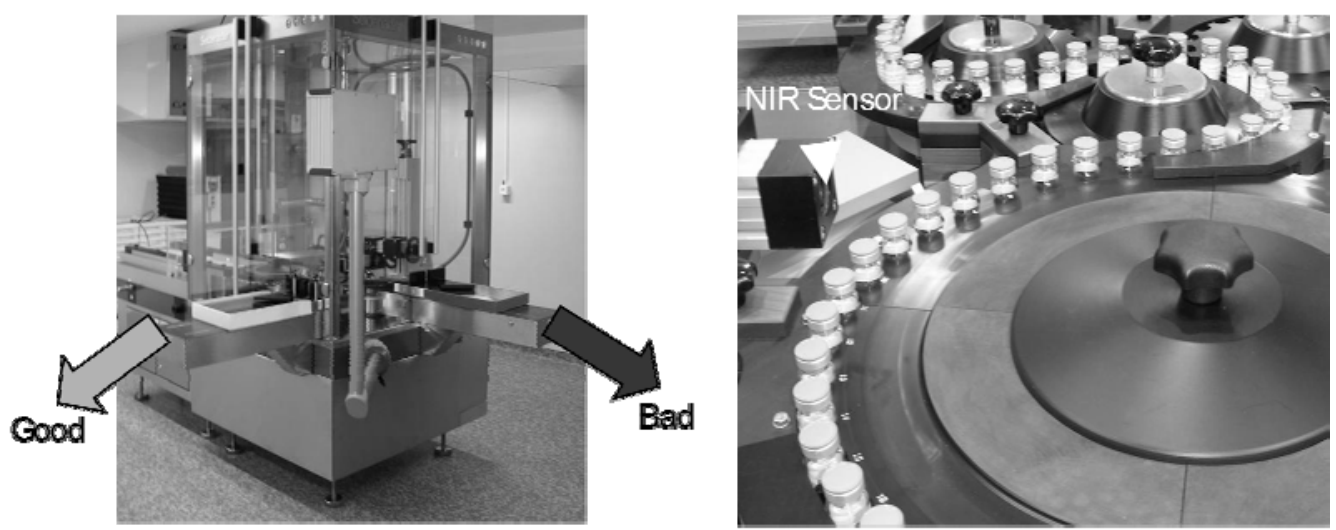

Figure 9.14. Near infrared (NIR) inspection machine for $100 \%$ on-line water content determination of lyophilised vials

\subsubsection{Content determination}

Many studies have been published during the last few years concerning the determination of the chemical content of compounds such as API, excipients or moisture in medicines. Potential samples for analysis can be of various forms like powders, granulates, tablets, liquids, gels, films or lyophilised vials [107-113].

A study was published comparing NIR spectrometers, such as FT-NIR, FTIR-PAS (Photoacoustic Spectroscopy), FTIR-ATR (Attenuated Total Reflectance), DRIFTS (Diffuse Reflectance Infrared Fourier Transform Spectroscopy) and FT-Raman for the determination of vitamin C in powders and solutions [114]. 
Chalus and co-workers presented different data pretreatments and regression methods for prediction models of API in low-dosage tablets [115]. An example of the quantitative analysis of API is presented in Figure 9.15 where the results of the PLS regression can be observed. Figure 9.15a presents the pretreated NIR spectra, while the selection of 13 factors from the cross validation is shown in Figure 9.15b. The results of the validation with reference and NIR predicted data are displayed in Figure 9.15c, and the statistics of the method in Figure 9.15d [116].
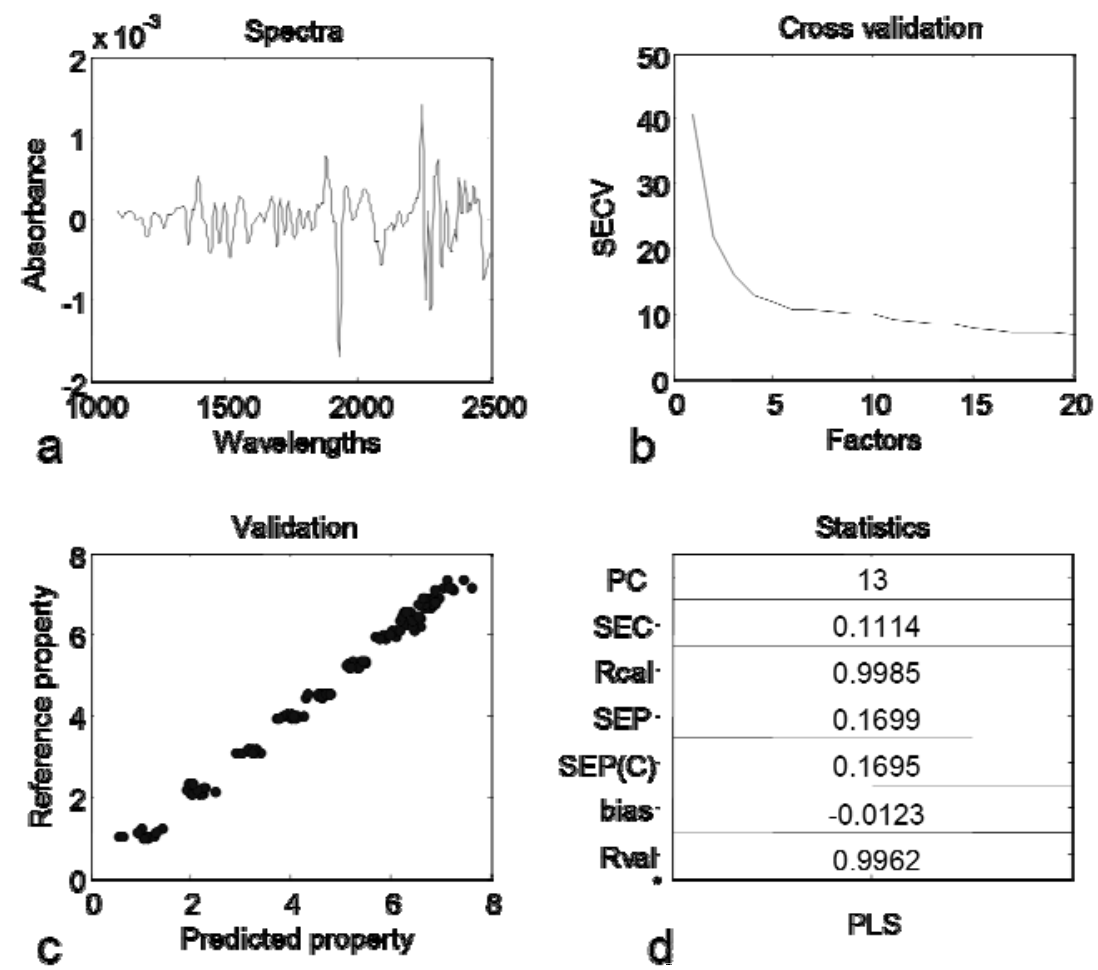

Figure 9.15. Determination of API content by NIRS (range: 1 - $8 \mathrm{mg}$ API/tablet)[116]. PC - principal component; SEC - standard error of calibration; SEP(C) - SEP with bias correction; API - active pharmaceutical ingredient; NIRS - near infrared spectroscopy

An increasing number of papers are published about the use of NIRS for the follow-up of the tablet production process, from the raw materials to the final product, with tablets being coated or not or packaged or not $[74,106,117,118]$. Successful implementation during early stage formulation development was also presented by Li [119]. NIRS spectroscopy allows a $100 \%$ packaging check of tablets with a built-in PCA model to sort up to 12000 tablets per minute [120]. Many other quantitative applications are summarised in Table 9.3. 
Table 9.3. Examples of quantitative applications of NIRS.

\begin{tabular}{|c|c|c|c|c|}
\hline $\begin{array}{l}\text { Sample } \\
\text { form }\end{array}$ & Analyte & $\begin{array}{l}\text { Regression } \\
\text { Method* }^{*}\end{array}$ & Remarks $\dagger$ & Ref. \\
\hline \multirow{10}{*}{ Tablets } & Coating thickness & PLS & $\begin{array}{l}\text { Measurements are made directly in a fluidised } \\
\text { bed and the coating thickness is followed. }\end{array}$ & [121] \\
\hline & Coating thickness & PLS & $\begin{array}{l}\text { Tablets are composed of two different chemical } \\
\text { compositions. }\end{array}$ & [122] \\
\hline & Ibuprofen $800 \mathrm{mg}$ & PLS & $\begin{array}{c}\text { Tablets of } 7.6 \mathrm{~mm} \text { thickness have to be reduced } \\
\text { to } 3.6 \mathrm{~mm} \text {. The transmittance measurement is } \\
\text { thus usable on a dedicated device. }\end{array}$ & [123] \\
\hline & Metformin & $\begin{array}{l}\text { PLS } \\
\text { Linear } \\
\text { regression }\end{array}$ & $\begin{array}{l}\text { PLS appeared to be more accurate than single } \\
\text { wavelength regression. }\end{array}$ & [124] \\
\hline & Caffeine & PLS & The range of caffeine content is $0-100 \% \mathrm{~m} / \mathrm{m}$. & {$[125]$} \\
\hline & Steroid & PLS & $\begin{array}{l}\text { Transmittance of the tablets is measured. The } \\
\text { SEP allowed the use of NIRS for the assay of } \\
\text { tablets for batch release. }\end{array}$ & [126] \\
\hline & Gemfibrozil & PLS & $\begin{array}{c}\text { Tablets of two related preparations are identified } \\
\text { by a classification model. Their content is } \\
\text { predicted for } 751 \mathrm{mg} / \mathrm{g} \text { and } 810 \mathrm{mg} / \mathrm{g} \\
\text { formulation. }\end{array}$ & [127] \\
\hline & Paracetamol & MLR, PLS & $\begin{array}{l}\text { Two wavelength selection modes were tried for } \\
\text { the MLR. }\end{array}$ & [128] \\
\hline & Paracetamol & MLR & The MLR model is computed on two wavelengths. & {$[129]$} \\
\hline & Acetylsalicylic acid & PLS & $\begin{array}{l}\text { This study assays acetylsalicylic acid in three dif- } \\
\text { ferent formulations: only API, API combined with } \\
\text { vitamin C, or with vitamin C and paracetamol. } \\
\text { Measurements are performed in reflectance and } \\
\text { transmittance on intact tablets and reflectance on } \\
\text { milled tablets. }\end{array}$ & {$[130]$} \\
\hline \multirow{3}{*}{$\begin{array}{l}\text { Tablets } \\
\text { Powders }\end{array}$} & $\begin{array}{l}\text { Paracetamol } \\
\text { Amantadine } \\
\text { Hydrochloride } \\
\end{array}$ & ANN & $\begin{array}{l}\text { Assays of paracetamol and amantadine hydroxide } \\
\text { are determined by an ANN model. Models are } \\
\text { based on tablets and powders. }\end{array}$ & [131] \\
\hline & Diphenhydramine & PLS & $\begin{array}{l}\text { Tablets of diphenhydramine are measured in } \\
\text { reflectance and transmittance. Their milled form } \\
\text { is measured in reflectance. Results are } \\
\text { comparable for the three kinds of measurements. }\end{array}$ & [132] \\
\hline & Mirtazapine & PLS & $\begin{array}{l}\text { The PLS model for determination of content is } \\
\text { based on lab powder samples and production } \\
\text { tablets. The first factors of the model had to be } \\
\text { excluded and the final selected model used } 4 \\
\text { factors. }\end{array}$ & [87] \\
\hline \multirow[t]{2}{*}{ Powder } & $\begin{array}{l}\text { Paracetamol } \\
\text { Diphenhydramine } \\
\text { Hydrochloride } \\
\text { Caffeine }\end{array}$ & ANN, PLS & $\begin{array}{l}\text { The study compares different pretreatments and } \\
\text { PLS to ANN. ANN improves the results compared } \\
\text { to classical PLS. }\end{array}$ & [133] \\
\hline & Amylose & Peak ratio & The computed model presents a RMSEP of $1.2 \%$. & {$[134]$} \\
\hline $\begin{array}{l}\text { Powder } \\
\text { Granulate }\end{array}$ & $\begin{array}{l}\text { Ferrous lactate } \\
\text { Dihydrate }\end{array}$ & PLS & $\begin{array}{l}\text { The concentration range was } 650-850 \mathrm{mg} / \mathrm{g} \text {. } \\
\text { Identification is first performed on the samples. } \\
\text { The lab samples are powders while production } \\
\text { ones are granulates and both are included in the } \\
\text { model. }\end{array}$ & [135] \\
\hline
\end{tabular}




\begin{tabular}{ccccc}
\hline $\begin{array}{c}\text { Sample } \\
\text { form }\end{array}$ & Analyte & $\begin{array}{c}\text { Regression } \\
\text { Method* }\end{array}$ & Remarks $\dagger$ & Ref. \\
\hline $\begin{array}{c}\text { Lyophilised } \\
\text { samples }\end{array}$ & Water & PLS & $\begin{array}{c}\text { Two PLS models are built, the first one for water } \\
\text { content of } 1-40 \% \text { w/w and the second one for } \\
\text { content between } 1 \text { and } 10 \% \text { w/w. }\end{array}$ & {$[136]$} \\
\hline $\begin{array}{c}\text { Extruded } \\
\text { film }\end{array}$ & Clotrimazole & PLS & $\begin{array}{c}\text { The drug content range was 0-20\% in a hot melt } \\
\text { extruded film of polyethylene oxide. }\end{array}$ & [137] \\
\hline $\begin{array}{c}\text { Translucent } \\
\text { gel }\end{array}$ & Ketoprofen & MLR, PLS & $\begin{array}{c}\text { MLR is preferred to PLS because it gives a good } \\
\text { idea of the ability of NIR to predict content. }\end{array}$ & [138] \\
\hline
\end{tabular}

* Partial least squares (PLS); multiple linear regression (MLR); artificial neural network (ANN)

† Near infrared spectroscopy (NIRS); active pharmaceutical ingredient (API); root mean square error of prediction (RMSEP)

\subsection{ON-LINE CONTROL BY MEANS OF NIRS}

\subsubsection{Powder Blending}

The blending of API with excipients is a critical step in the manufacturing of pharmaceutical solids. Without a homogenous blend it is impossible to get uniform doses with the right content of API in the final product. However, the determination of homogeneity is problematic. Currently, samples are mostly removed from the blender and analyzed by conventional methods like HPLC or UV/VIS-spectroscopy. The API distribution is thus determined and the homogenous distribution of the excipients assumed if the API is homogenously distributed. Moreover, the sampling often changes the powder distribution and is consequently the source of significant sampling errors. Classical methods are besides destructive, time and cost consuming, labor intensive, require solvents and are responsible for long cycle times as they are performed off-line. Therefore, the use of a fast, non-destructive method is advisable. NIRS offers these advantages and enables the analysis of all the compounds of a powder mixture. On-line or in-line application is possible for homogeneity (Figure 9.16) and end point determination.

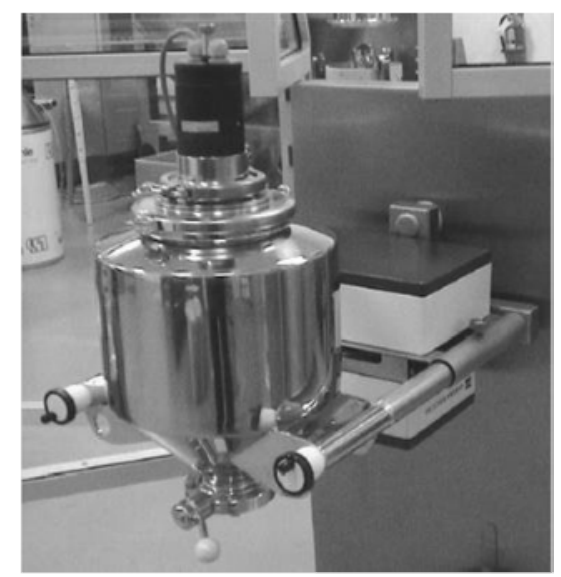

Figure 9.16. Implementation of an on-line near infrared (NIR) spectrometer in solid development for blending monitoring 
Numerous studies have been carried out to explore the use of NIRS for powder blending control. The fact that NIRS has great potential in this application has been shown by Wargo and Drennen [139-142]. Cho and co-workers dealt with the effective mass that is sampled by NIR fibre-optic reflectance probes in blending processes and demonstrated that the sampled mass met FDA requirements [143]. Hailey and co-workers showed that by using a fibre-optic reflectance probe it is possible, either in a y-cone or blender, to use NIRS for in-line blend analysis [144]. Sekulić and co-workers also evaluated NIRS for on-line monitoring of powder blending processes by using a fibre-optic reflectance probe and showed its feasibility with a model-free approach [145]. NIR imaging was used by El-Hagrasy and co-workers who demonstrated the possibility of using it for on-line blending control. However, they pointed out the fact that multiple sampling points are necessary for correct process control [21]. Sekulić and co-workers focused on qualitative approaches of blend evaluation in a study using a small blender and a reflectance fibre optic probe. Different blends were produced, monitored via NIRS and different mathematical pre-processing performed on the resulting data [146]. Skibsted and co-workers presented a qualitative and quantitative method and control charts, with which they were able to monitor the homogeneity of a blend [147]. The use of NIRS for the quantification of the drug content has also been used by Popo and co-workers. However, they measured samples obtained by streamsampling instead of taking spectra directly in the blender [148]. Berntsson and coworkers described the quantitative in-line monitoring in a mixer, both in the lab and at the production scale. With high speed sampling, average content and distribution of the mixture content were assessed [149].

Figure 9.17 presents an example of a blend process monitoring of API using the moving block method. In Figure 9.18, a quantitative determination of API is performed during the blend process. Since 2007, a large number of publications have been written about NIRS and the blend process [150-160]. The use of this spectroscopy for blend monoriting has therefore been fully demonstrated.

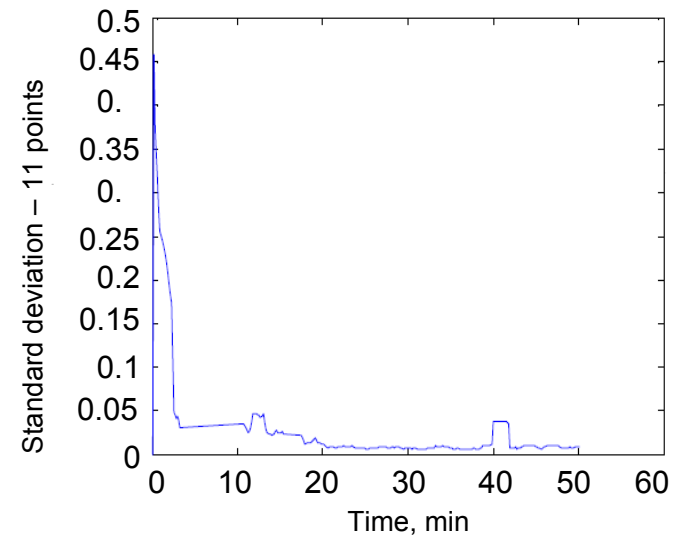

Figure 9.17. Moving block standard deviation at a specific wavelength of active pharmaceutical ingredient (API) during the blend process 


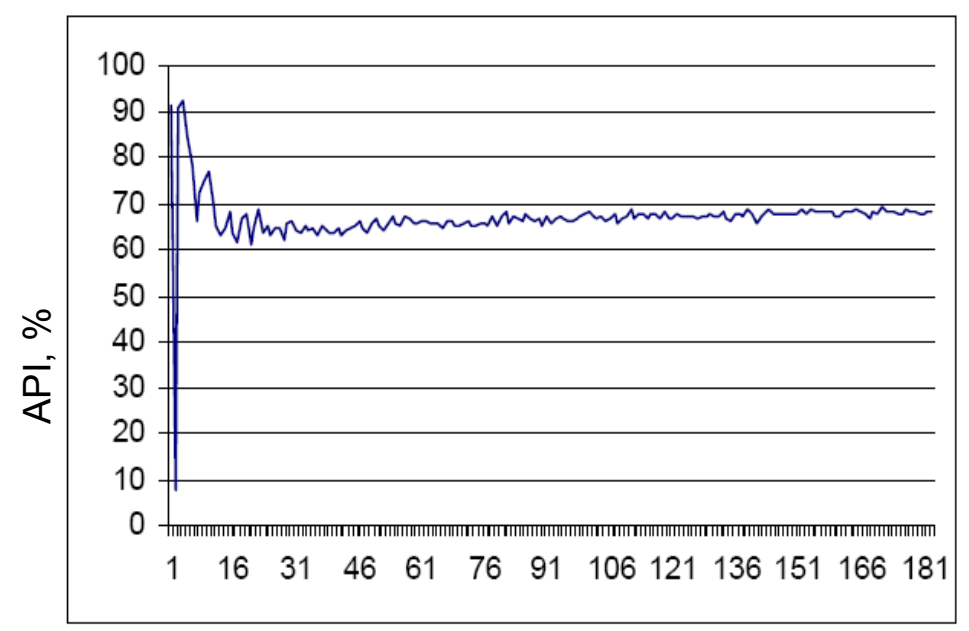

Rotations

Figure 9.18. On-line quantitative determination of the active pharmaceutical ingredient (API) content during the blend process.

\subsubsection{Granulation}

In many cases, powder blending is followed by a granulation step, which is often necessary for tablet compression or capsule filling. These are produced either by dry granulation, like roller compaction, wet granulation, such as fluid bed spray, or high shear mixer granulation. The moisture content of granulates is important for the rest of the process as it influences their properties and, for example, the hardening of tablets during the storage. Classical measurement methods such as infrared dryers for moisture content determination require time and consequently slow down the manufacturing on the development process. As NIRS can be performed in real time, the process might be monitored more efficiently, resulting in greater process reliability and optimized product characteristics. NIR can be used during the process optimization step. Rantanen and co-workers used NIR-reflectance spectroscopy for in-line moisture content determination in fluidised bed granulation. They followed spraying and drying phases by NIRS and were able to determine drying end points $[102,161]$ and the effects of binder and particle size on moisture determination [162]. A non-linear calibration model was developed with a combination of NIRS and other process measurements [163]. Frake and co-workers applied in-line NIRS to a fluidised bed granulation to control the granule moisture content and changes in particle size [164]. Findlay and co-workers showed that this spectroscopy enables the control of a fluidised bed granulation. They determined the drying end and time points when binder addition should be stopped [100]. Gupta and co-workers determined content uniformity, moisture content and strength of compacts [165]. Ultimately, NIRS is a useful tool for the monitoring of the different phases of the granulation process and determination of particule size and API content [166]. The granulation rate can be estimated by NIRS as well [167]. 


\subsubsection{Drying}

Drying is most of the time another critical step in the production of medicines. It is used at different stages of the process, such as granulation or lyophilisation. As the $\mathrm{O}-\mathrm{H}$ bands are characteristic by NIRS, the first on-line applications of this spectroscopy were mainly for the monitoring of drying processes. In Figure 9.19, the evolution of NIR spectra during a drying process can be observed. Indeed at the position of the water bands, especially at $1950 \mathrm{~nm}$, the analyzed spectra present a variation of the intensity, which can be directly correlated to the moisture of the samples.
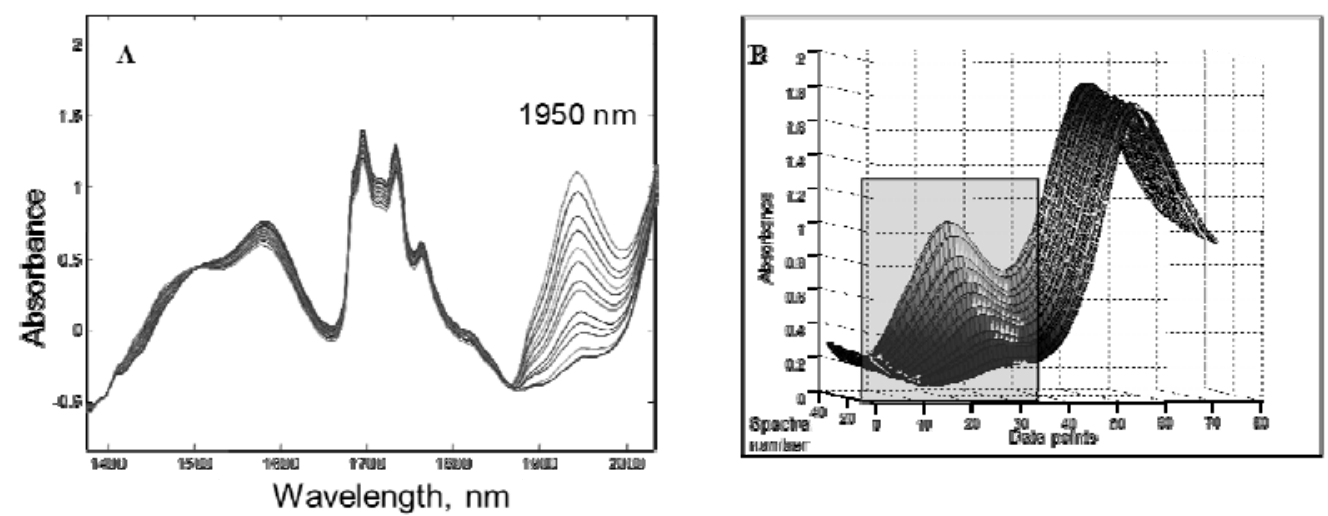

Figure 9.19. On-line water content determination during the active pharmaceutical ingredient (API) drying step. In Figure 9.19A, the analyzed spectra present a variation of the intensity of the water peak at $1950 \mathrm{~nm}$. The differences between the spectra are highlighted in Figure 9.19B

Brülls and co-workers presented the possibility of following the transition in the cake during a freeze drying step. The measurement, performed by introducing a fibre optic probe in a vial, showed a good correlation with the classical method [168]. Sukowski and Ulmschneider studied the analysis of $100 \%$ production vials directly in-line [169]. The use of NIR for the drying process was validated and transferred by Peinado and co-workers [170].

\subsubsection{Crystallinity and polymorphism}

During the drying phase of wet granulation, polymorphic changes can occur in an API or in some of the excipients. The polymorphic changes of glycine involve important modifications in the hydrogen bonding of crystals. They were quantified by Davis and co-workers with NIRS during wet granulation [171].

Crystallisation may also be followed in an earlier step, i.e. the production of an API. Févotte and co-workers used a fibre optic probe to monitor crystallisation by NIRS. This method showed the possibility of using this type of spectroscopy to follow the API crystallisation on-line [98]. 


\subsubsection{Coating}

One of the last steps in the preparation of a drug may involve the coating of some tablets or granulates. It is important to ensure the integrity and good quality of the drug because coating may influence the release of the drug or assure its stability. NIR diffuse reflectance spectroscopy was used with a fibre optic probe to determine the film coating thickness of pellets by means of a PLS model. The probe was inserted on a side port of the fluidized bed reactor, and located vertically to the pellet bed [121]. In the case of Pérez-Ramos and co-workers who dealt with tablets, the probe was placed directly in the coating pan for diffuse reflectance measurement. A univariate model was used, which followed the decrease and increase of specific bands of a core compound and the coating, respectively [172]. NIR and Raman spectroscopy were used simultaneously during the fluid bed pellet coating process by Dogomolov and co-workers [173]. The coating thickness of pellets $[174,175]$ or tablets [176] was proven to be measurable by NIRS.

\subsubsection{Biotechnology}

A new field of application for NIRS, following trends in biotechnological manufacturing processes, has lately emerged in the pharmaceutical field. This tool, combined with chemometrics, enables cell culture monitoring, for instance, as in the system presented in Figure 9.20.

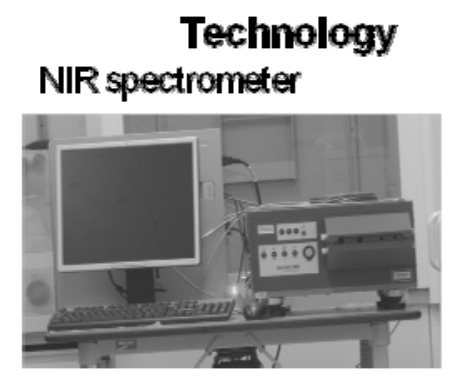

\section{NIR Probe (transflexion 1mm) in a bioreactor}

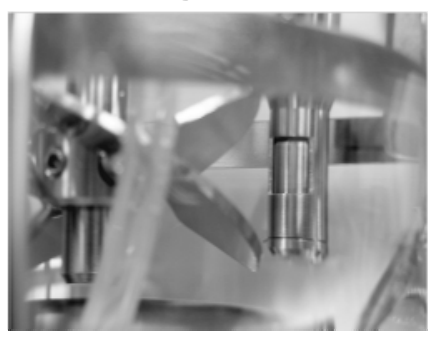

Goal

Online monitoring of cell culture parameters (Gucose, Lactate, Titer, Vability, ...)

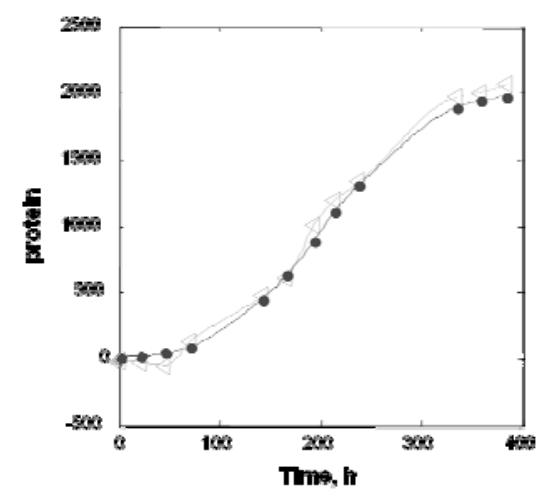

Figure 9.20. Near infrared (NIR) and cell culture monitoring Probe and instrumentation 
In 2002 and 2003, Arnold and co-workers presented the acquisition, calibration, validation and implementation of the fermentation process including controlling and monitoring. In these studies, the authors managed to collect NIR spectra in transmittance and reflectance modes. For the direct on-line or in-line implementation, development was advised because the transfer from at-line to in-line analyses would be a challenge [177,178]. Cimander and Mandenius in 2002 applied the methodology on the fermentation of Escherichia coli to produce antibiotics. Spectra were acquired with an immersion probe and PCA and PLS were used as chemometric tools. Models to track the amounts of biomass, tryptophan, phosphate, glucose and acetate were developed and validated [179]. Vibrio cholerae fermentations used to produce toxins or plasmids were measured by Navratil and co-workers in 2004. NIR spectra were acquired with a fibre optic probe and PLS computed to develop calibrations of biomass, glucose and acetate. Interference problems that occurred when applying the chosen models in the bioreactor were discussed and corrected. Finally, the authors applied NIR prediction models in the production [180]. In 2003, Tamburini and co-workers tried to monitor the fermentation of Staphylococcus and Lactobacillus. Spectra were also acquired with a fibre optic probe and PLS regression was applied to develop models for glucose, lactic acid, acetic acid and biomass. These models were then used for automatic control [181]. The determination of biomass, glucose, lactic acid and acetic acid during fermentations of Staphylococcus xylosus was performed by Tosi and co-workers. Models were developed by PLS for glucose, biomass, lactic acid and acetic acid. The SEC and SEP were satisfactory and the models were then applied to other microorganisms in the same medium [182]. The study of Yeung and co-workers [183] compared two strategies for the preparation of calibration samples of a Saccharomyces cerevisiae bioprocess. PLS was applied to the NIR spectra to obseve cell debris, protein and RNA. The calibration models selected according to the SEP values were finally validated.

Traditionally, many fermentation products come from microbial bioprocesses. However, lately, mammalian and insect cell cultivations were also exploited for the high-cost products they can be engineered to produce. Arnold and coworkers [184] developed a method to monitor mammalian cell cultivation. NIR spectra were acquired with an immersion probe and models were constructed for glucose, lactate, glutamine and ammonia. External and internal validation was performed. The monitoring of insect cell culture was already developed by Riley and co-workers in 1996 [185]. Calibration models were established for glutamine and glucose with PLS and the models could be used for high concentrations. Studies about cell culture media were made Lewis and co-workers in 2000 [186] and Jung and co-workers in 2002 [187]. In the first study, the authors developed models to predict glucose production using PLS. The results were compared according to the SEC, SEP and mean percent error (MPE) and the best model for the control of culture was retained. This demonstrated the ability of NIRS to monitor on-line fermentations and cell cultures. In the second study, the system was coupled with a lab-system to provide a real-time spectral background 
reference. Smoothing and PLS were applied to develop calibration models for glucose and lactate.

During the last 3 years, the number of publications dealing with fermentation or cell culture monitoring has significantly increased [188-193], some of them being of very good quality like the report by Henriques and Buziol [194]. NIR can therefore be used for the development of bioprocess.

\subsection{APPLICATIONS OF NIR SPECTRAL IMAGING}

\subsubsection{General use of NIR chemical imaging for pharmaceutical applications}

The chemical compound homogeneity is an important issue for the development of pharmaceutical solids. A classical NIR spectrometer integrates spatial information $[130,195,196]$. However, the use of a mean spectrum on the surface can be a drawback in solid form analysis. On the contrary, hyperspectral imaging provides information that is spatial and spectral, and both qualitative and quantitative. It can map chemical compound distribution and determine particle size. In the pharmaceutical industry, it is for instance important to map the distribution of APIs and excipients in a tablet as this reveals physical interaction between components and helps solve homogeneity issues. This explains the increasing number of spectroscopic imaging studies on the visualization of chemical component homogeneity [21,197-201]. The method was applied to quality control and to process problems affecting pharmaceutical tablets: dissolution, polymorph distribution, moisture content determination, API localization and characterization, counterfeits' detection, blending, and granulation.

Spectral imaging is a complex and multidisciplinary field. The introduction of new detectors is making its use increasingly powerful and attractive. It has proven its potential for qualitative pharmaceutical analyses and can be used when spatial information becomes relevant for an analytical application. Even if online applications and regulatory method validation require further study, the potential contribution of imaging to quality control and PAT needs no further demonstration. A detailed overview of the pharmaceutical applications of NIR imaging and chemometric tools for image analysis is available in several reviews $[202,203]$. Only two examples will be discussed here.

\subsubsection{Tablet composition analysis with NIR imaging}

An example of the reconstruction of a tablet by NIR spectral imaging is presented below. A tablet was cut lengthwise with a trimmer to get a plane surface and the coating was then removed. The sample and references were analyzed using a chemical imaging NIR spectrometer (Sapphire ${ }^{\mathrm{TM}}$, Malvern) with the following acquisition parameters: detector size of $320 \times 256$ array, spectral range of $1100-2450 \mathrm{~nm}$ and a spatial resolution of $40 \mu \mathrm{m} /$ pixel. The acquisition lasted about 5 minutes. 
After the pretreatment of the spectra with a second derivative, wavelengths were selected to give contrast images and display the localization of mannitol, API and crospovidone with NIR images at specific wavelengths (Figure 9.21).

After the aforementioned steps were performed, tablet reconstruction by NIR imaging was then possible. This highlights the main advantage of imaging: the large area of analysis. The images are indeed more representative of the sample than a mean spectrum of an entire tablet.

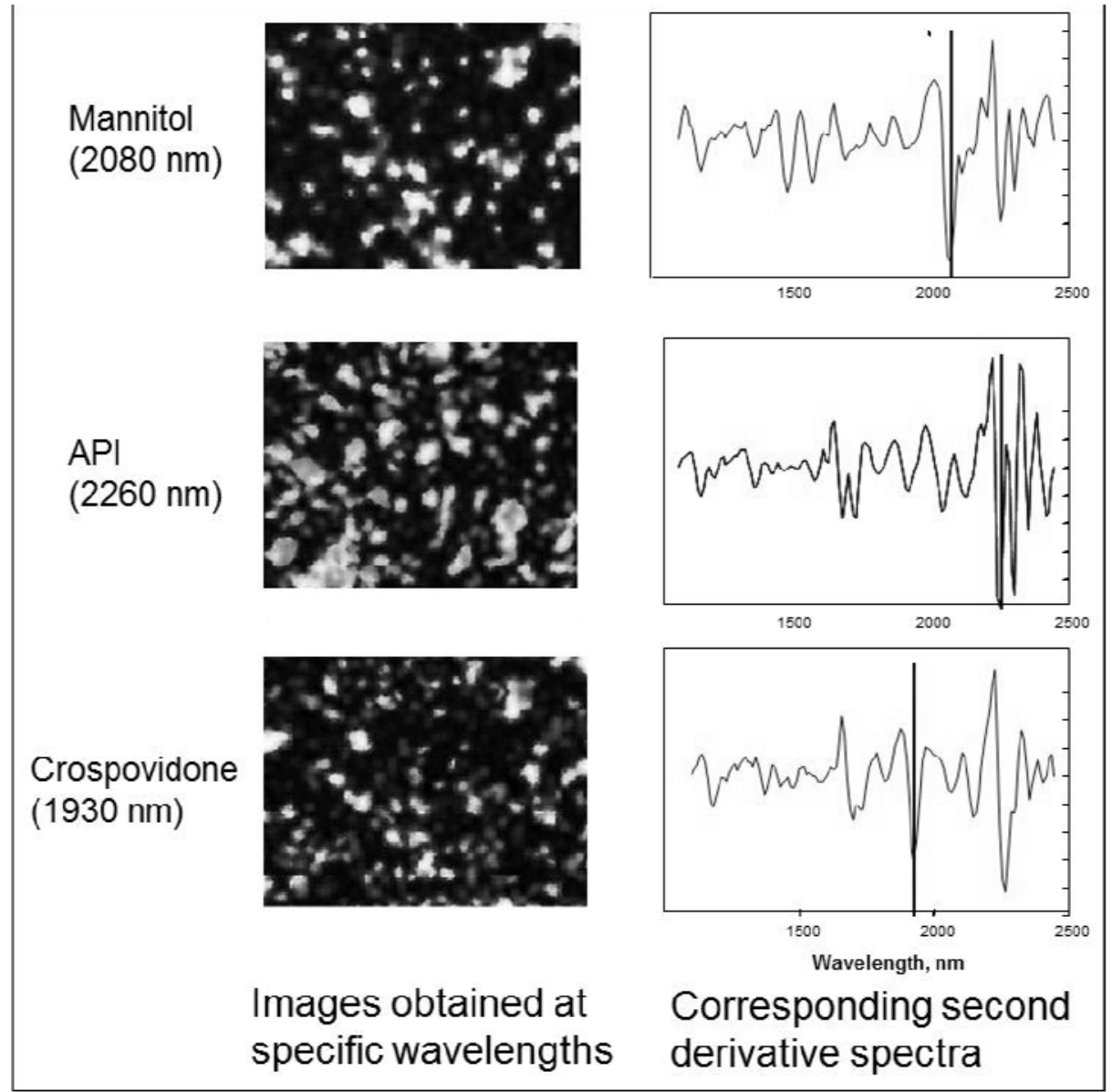

Figure 9.21. Use of multivariate curve resolution-alternating least squares (MCR-ALS) in order to obtain distribution maps - near infrared (NIR) imaging [1]

API - active pharmaceutical ingredient

\subsubsection{Application of NIR imaging to process optimization}

The aim of this study was to use NIR imaging to solve granulation issues in a new formulation development. Undesired powder agglomerations were indeed developed during the granulation step and imaging was applied to characterize the structure. 
The measured sample contained API, starch, Avicel ${ }^{\circledR}$, crospovidone, and sodium lauryl sulfate. The sample and references were analyzed by NIR imaging (Malvern system) with 20 co-adds on a spectral range of $1100-2450 \mathrm{~nm}$. The full image size was $320 \times 256$ pixels (or $4.1 \times 3.3 \mathrm{~mm}$ ). The images were interpreted and sample raw materials mapped using PLS classification with five loadings. This was based on the reference spectra of starch, API, Avicel ${ }^{\circledR}$, crospovidone, and sodium lauryl sulfate.

The PLS model allowed the identification all five chemical compounds. The PLS multivariate analysis showed that the core contained Avicel ${ }^{\circledR}$ and API and starch and crospovidone in the periphery (Figure 9.22). Thanks to this information, a solution to the granulation issue could be found consisting of the addition of a premixing step to avoid agglomeration. NIR imaging proved to be useful in the improvement of process understanding. In our case, the powder agglomeration was heterogeneous and the layers had high excipient content, making it possible to apply supervised classification.

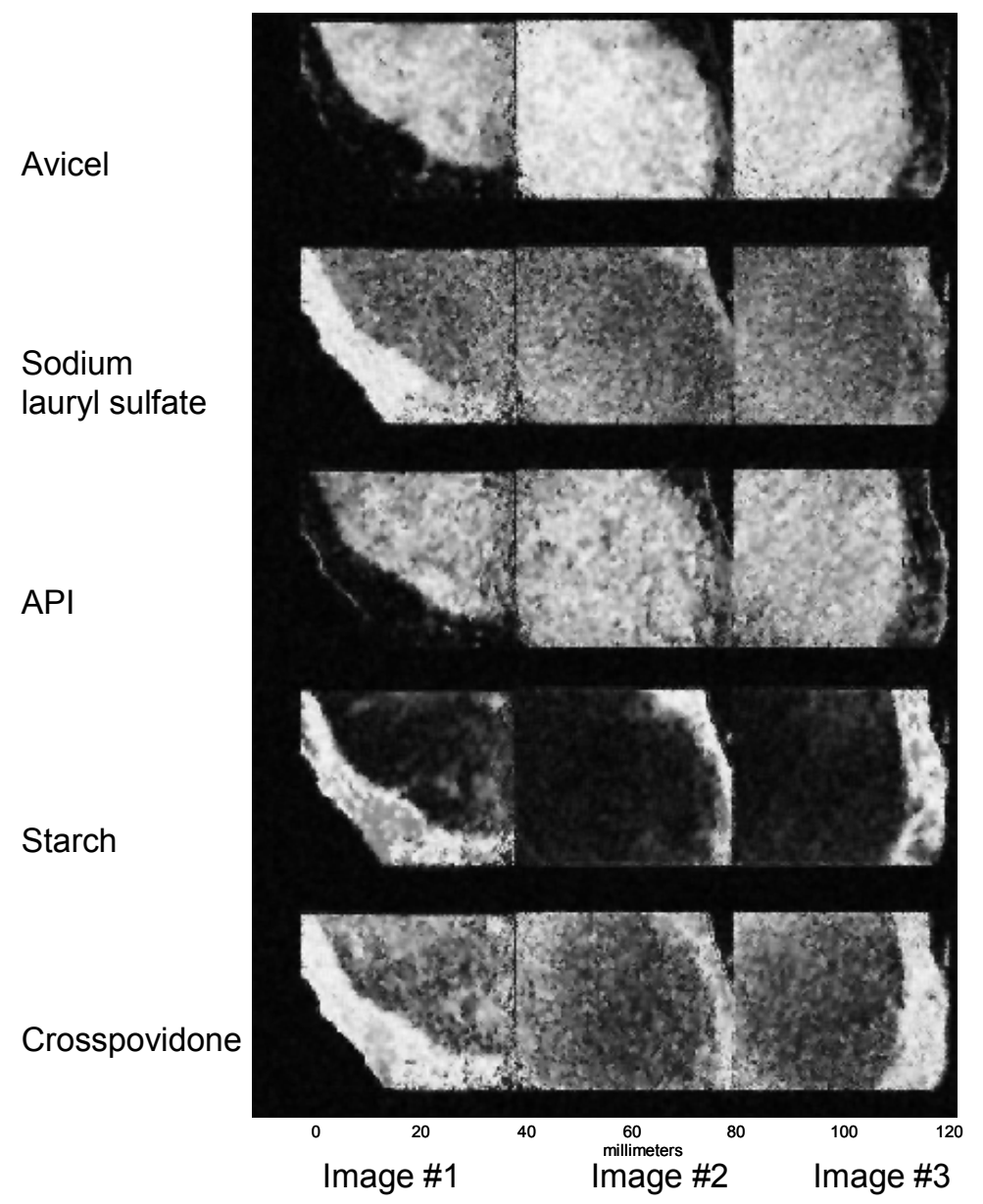

Figure 9.22. Images obtained by classification methods - near infrared (NIR) imaging [1]. API - active pharmaceutical ingredient 


\subsection{CONCLUSION}

The potential of NIRS for process development needs no further demonstration, as NIRS is a powerful way to discriminate pharmaceutical compounds. This method can be used qualitatively to detect, identify and qualify as well as control raw materials and final products. Additionally, it is a suitable tool for the classification and quantification of pharmaceutical samples. NIRS is moreover a potentially precious diagnostic technique in process trouble-shooting and can provide spectral profiles of pharmaceutical products. Besides, it can support the development of new processes and drug discovery. NIRS can be applied across the pharmaceutical production process in chemistry, biotechnology and galenic fields $[204,205]$. The success of this analytical technique relies on key advantages [46]. As previously observed, NIRS is influenced by the chemical and the physical properties of the samples. This spectroscopy requires limited or no sample preparation and is non-destructive. Moreover, the measurement is fast with performance in less than a second for on-line applications and NIR frequencies are transmitted through glass. Other vibrational techniques like Raman spectroscopy [206] and mid-IR should be mentioned. These techniques can also be applied successfully to solve pharmaceutical issues in order to support the development of new drugs and new processes. Finally, NIR imaging systems were developed in recent years. Namely, a hyper-spectral imaging spectrometer records simultaneously spectra and spatial information of samples. NIR imaging [207] completes NIR spectroscopy and is used when spatial distribution is an important issue of the analysis.

\section{REFERENCES}

1. Y. Roggo, M. Ulmschneider. Chemical Imaging and Chemometrics: Useful Tools for Process Analytical Technology. Wiley, 2009, Chapter 4.3.

2. W. Chew, P. Sharratt. Trends in process analytical technology. Analytical Methods 2(10) (2010) 1412-1438.

3. T. Herdling, D. Lochmann. Implementation of Process Analytical Technology (PAT) in the production of solid dosage. Implementierung von Process Analytical Technology (PAT) in der solida-produktion. Pharmazeutishe Industrie 72(3) (2010) 402-408.

4. S.H. Tabasi, R. Fahmy, D. Bensley, C. O`Brien, S.W. Hoag. Quality by design, part II: Application of NIR spectroscopy to monitor the coating process for a pharmaceutical sustained release product. Journal of Pharmaceutical Sciences 97(9) (2008) 4052-4066.

5. K.H. Norris. History of NIR. Journal of Near Infrared Spectroscopy 4(1-4) (1996) 31-37.

6. Y. Roggo, L. Duponchel, J.-P. Huvenne. Quality evaluation of sugar beet (beta vulgaris) by near-infrared spectroscopy. Journal of Agricultural and Food Chemistry 52(5) (2004) 1055-1061. 
7. P. Williams, K. Norris, Editors, Near-Infrared Technology in the Agricultural and Food Industries. American Association of Cereal Chemists, St. Paul, MN (1987) 143-167.

8. M.S. Larrechi, M.P. Callao. Strategy for introducing NIR spectroscopy and multivariate calibration techniques in industry. TrAC Trends in Analytical Chemistry 22(9) (2003) 634-640.

9. M. Blanco, S. Maspoch, I. Villarroya, X. Peralta, J.M. González, J. Torres. Geographical origin classification of petroleum crudes from near-infrared spectra of bitumens. Applied Spectroscopy 55(7) (2001) 834-839.

10. H.P.R. Aenugu, D.S. Kumar, S.N. Parthiban, S.S. Ghosh, D. Banji. Near infra red spectroscopy- An overview. International Journal of ChemTech Research 3(2) (2011) 825-836.

11. Y. Roggo, P. Chalus, L. Maurer, C. Lema-Martinez, A. Edmond, N. Jent. A review of near infrared spectroscopy and chemometrics in pharmaceutical technologies. Journal of Pharmaceutical and Biomedical Analysis 44(3) (2007) 683-700.

12. J. Luypaert, D.L. Massart, Y. Vander Heyden. Near-infrared spectroscopy applications in pharmaceutical analysis. Talanta 72(3) (2007) 865-883.

13. D. Cozzolino. A review on the use of near infrared spectroscopy for plant analysis. Medicinal Plants - International Journal of Phytomedicines and Related Industries 2(1) (2010) 13-20.

14. E. Räsänen, N. Sandler. Near infrared spectroscopy in the development of solid dosage forms. Journal of Pharmacy and Pharmacology 59(2) (2007) 147-159.

15. L. Bokobza. Near infrared spectroscopy. Journal of Near Infrared Spectroscopy 6(14) (1998) 3-17.

16. D.A. Burns, E.W. Ciurczak. Handbook of Near-Infrared Analysis. $2^{\text {nd }}$ Edition, Marcel Dekker, 2001, p. 814.

17. S. Morimoto, W.F. McClure, D.L. Stanfield. Hand-Held NIR Spectrometry: Part 1: An Instrument Based upon Gap-Second Derivative Theory. Applied Spectroscopy 55(2) (2001) 182-189.

18. L.F. Capitán-Vallvey, A.J. Palma. Recent developments in handheld and portable optosensing-A review. Analytica Chimica Acta 696(1-2) (2011) 27-46.

19. K. Dégardin, Y. Roggo, P. Margot. Evaluation de spectromètres portables raman, infrarouge et proche infrarouge pour la détection de contrefaçons de médicaments. Spectra Analyse 276 (2010) 46-52.

20. E.N. Lewis, J.W. Schoppelrei, E. Lee, L.H. Kidder. Near-Infrared Chemical Imaging as a Process Analytical Tool. In: Process Analytical Technology, (Editor: K.A. Bakeev), Blackwell publishing Ltd., 2005, p. 187-225.

21. A.S. El-Hagrasy, H.R. Morris, F. D`Amino, R.A. Lodder, J.K. Drennen III. Nearinfrared spectroscopy and imaging for the monitoring of powder blend homogeneity. Journal of Pharmaceutical Sciences 90(9) (2001) 1298-1307.

22. L. Breiman, J.H. Friedman, R.A. Olsen, C.J. Stone. Classification and Regression Trees 1984. Wadsworth, 1983.

23. D.L. Massart, B.G.M. Vandeginste, L.M.C. Buydens, S. De Jong, P.J. Lewi, J. SmeyersVerbeke. Handbook of Chemometrics and Qualimetrics: Part A. Data Handling in Science and Technology, Vol. 20A. Elsevier, 1997. 
24. B.G.M. Vandeginste, D.L. Massart, L.M.C. Buydens, S. De Jong, P.J. Lewi, J. SmeyersVerbeke. Handbook of Chemometrics and Qualimetrics: Part B. Data Handling in Science and Technology. Elsevier, 1998.

25. B.K. Lavine. Chemometrics. Analytical Chemistry 72(12) (2000) 91-98.

26. M. Otto. Chemometrics Statistics and Computer Application in Analytical Chemistry. Wiley-VCH, 1999.

27. R.G. Brereton. Chemometrics Data Analysis for the Laboratory and Chemical Plant. John Wiley \& Sons, 2003.

28. D.L. Massart, B.G.M. Vandeginste, S.N. Deming, Y.Michotte, L. Kaufman. Chemometrics: a textbook. Journal of Chemometrics 2(4) (1988) 298-299.

29. M. Blanco, M.A. Romero, M. Alcala. Strategies for constructing the calibration set for a near infrared spectroscopic quantitation method. Talanta 64(3) (2004) 597-602.

30. M. Blanco, J. Coello, H. Iturriaga, S. Maspoch, C. de la Pezuela. Strategies for constructing the calibration set in the determination of active principles in pharmaceuticals by near infrared diffuse reflectance spectrometry. Analyst 122(8) (1997) 761-765.

31. J.H. Kalivas, Basis sets for multivariate regression. Analytica Chimica Acta 428(1) (2001) 31-40.

32. H.A.D. Filho, R.K.H. Galvão, M.C.U. Araújo, E.C. da Silva, T.C.B. Saldanha, G.E. José, C. Pasquini, I.M. Raimundo Jr., J.J.R. Rohwedder. A strategy for selecting calibration samples for multivariate modelling. Chemometrics and Intelligent Laboratory Systems 72(1) (2004) 83-91.

33. R.K.H. Galváo, M.C.U. Araujo, G.E. José, M.J.C. Pontes, E.C. Silva, T.C.B. Saldanha. A method for calibration and validation subset partitioning. Talanta 67(4) (2005) 736-740.

34. T. Naes, T. Isaksson, T. Fearn, T. Davies. Multivariate Calibration and Classification. NIR Publications, 2002.

35. M.C. Sarraguça, J.A. Lopes. The use of net analyte signal (NAS) in near infrared spectroscopy pharmaceutical applications: Interpretability and figures of merit. Analytica Chimica Acta 642(1-2) (2009) 179-185.

36. F. Been, Y. Roggo, K. Dégardin, P. Esseiva, P. Margot. Profiling of Counterfeit Medicines by Vibrational Spectroscopy. Forensic Science International 211(2011) 83-100.

37. D. González-Arjona, V. González-Gallero, F. Pablos, A.G. González. Authentication and differentiation of irish whiskeys by higher-alcohol congener analysis. Analytica Chimica Acta 381(2-3) (1999) 257-264.

38. L. Simon, M. Nazmul Karim. Probabilistic neural networks using Bayesian decision strategies and a modified gompertz model for growth phase classification in the batch culture of bacillus subtilis. Biochemical Engineering Journal 7(1) (2001) 41-48.

39. F. Marini, F. Balestrieri, R. Bucci, A.D. Magri, A.L.Magri, D. Marini. Supervised pattern recognition to authenticate Italian extra virgin olive oil varieties. Chemometrics and Intelligent Laboratory Systems 73(1) (2004) 85-93.

40. International Conference on Harmonisation Q2A, ICH Q2A: Text on Validation of Analytical Procedures. FDA Federal Register 60(96) (1995) 11260.

41. International Conference on Harmonisation Q2B, ICH Q2B: Validation of Analytical Procedures: Methodology. FDA Federal Register 62(96) (1997) 27463-27467. 
42. International Conference on Harmonisation Q6A, ICH Q6A, Specifications: Test Procedures and Acceptance Criteria for New Drug Substances and New Drug Products: Chemical Substances. FDA Federal Register 65(251) (2000) 83041-83063.

43. International Conference on Harmonisation Q7A, ICH Q7A: Good Manufacturing Practice Guide for Active Pharmaceutical Ingredients. 66(186) (2001) 4902849029.

44. EMEA, European Pharmacopeia (2011).

45. J. Märk, M. Andre, M. Karner, C.W. Huck. Prospects for multivariate classification of a pharmaceutical intermediate with near-infrared spectroscopy as a process analytical technology (PAT) production control supplement. European Journal of Pharmaceutics and Biopharmaceutics 76(2) (2010) 320-327.

46. C. van der Vlies. The use of NIR spectrometry in the pharmaceutical QC laboratory. European Journal of Pharmaceutical Sciences 2(1-2) (1994) 79-81.

47. H.R.H. Ali, H.G.M. Edwards, I.J. Scowen. Noninvasive in situ identification and band assignments of some pharmaceutical excipients inside USP vials with FT-nearinfrared spectroscopy. Spectrochimica Acta - Part A: Molecular and Biomolecular Spectroscopy 72(4) (2009) 890-896.

48. G. Chen, F. Kamal, J. Phillips. A comprehensive strategy to develop and implement a multi-site NIR method for identification of raw materials. American Pharmaceutical Review 12(6) (2009) 54-61.

49. M. Ulmschneider, G. Barth, E. Trenka. Building transferable cluster calibrations for the identification of different solid excipients with near-infrared spectroscopy. Pharmazeutische Industrie 62(5) (2000) 374-376.

50. M. Ulmschneider, E. Penigault. Assessing the transfer of quantitative NIR calibrations from a spectrometer to another one. Analusis 28(1) (2000) 83-87.

51. M. Ulmschneider, E. Penigault, Non-invasive confirmation of the identity of tablets by near-infrared spectroscopy. Analusis 28(4) (2000) 336-346.

52. M. Ulmschneider, A. Wunenburger, E. Penigault, Using near-infrared spectroscopy for the noninvasive identification of five pharmaceutical active substances in sealed vials. Analusis 27(10) (1999) 854-856.

53. N.K. Ebube, N.K. Ebube, S.S. Thosar, R.A. Roberts, M.S. Kemper, R. Rubinovitz, D.L. Martin, G.E. Reier, T.A. Wheatley, A.J. Shukla. Application of near-infrared spectroscopy for nondestructive analysis of Avicel powders and tablets. Pharmaceutical Development and Technology 4(1) (1999) 19-26.

54. K. Kramer, S. Ebel. Application of NIR reflectance spectroscopy for the identification of pharmaceutical excipients. Analytica Chimica Acta 420(2) (2000) 155-161.

55. K. Kreft, B. Kozamernik, U. Urleb. Qualitative determination of polyvinylpyrrolidone type by near-infrared spectrometry. International Journal of Pharmaceutics 177(1) (1999) 1-6.

56. A.O. Kirdar, G. Chen, J. Weidner, A.S. Rathore. Application of near-infrared (NIR) spectroscopy for screening of raw materials used in the cell culture medium for the production of a recombinant therapeutic protein. Biotechnology Progress 26(2) (2010) 527-531.

57. X.-M. Chong, C.-Q. Hu, Y.-C. Feng, H.-H. Pang. Construction of a universal model for non-invasive identification of cephalosporins for injection using near-infrared diffuse reflectance spectroscopy. Vibrational Spectroscopy 49(2) (2009) 196-203. 
58. L. Alvarenga, D. Ferreira, D. Altekruse, J.C. Menezes, D. Lochmann. Tablet identification using near-infrared spectroscopy (NIRS) for pharmaceutical quality control. Journal of Pharmaceutical and Biomedical Analysis 48(1) (2008) 62-69.

59. R. De Maesschalck, T. Van den Kerkhof. Implementation of a simple semiquantitative near-infrared method for the classification of clinical trial tablets. Journal of Pharmaceutical and Biomedical Analysis 37(1) (2005) 109-114.

60. P. de Peinder, M.J. Vreedenbregt, T. Visser, D. de Kaste. Detection of Lipitor ${ }^{\circledR}$ counterfeits: A comparison of NIR and Raman spectroscopy in combination with chemometrics. Journal of Pharmaceutical and Biomedical Analysis 47(4-5) (2008) 688-694.

61. Y.C. Feng, X.B. Zhang, C.Q. Hu. Construction of identification system for noninvasive analysis of macrolides tablets using near infrared diffuse reflectance spectroscopy. Journal of Pharmaceutical and Biomedical Analysis 51(1) (2010) 12-17.

62. R. Kalyanaraman, G. Dobler, R. Rubick, Portable spectrometers for pharmaceutical counterfeit detection. American Pharmaceutical Review 13(3) (2010) 38-45.

63. A.C. Moffat, S. Assi, R.A. Watt. Identifying counterfeit medicines using near infrared spectroscopy. Journal of Near Infrared Spectroscopy 18(1) (2010) 1-15.

64. A.J.O. Neil, R.D. Jee, G. Lee, A. Charvill, A.C. Moffat. Use of a portable near infrared spectrometer for the authentication of tablets and the detection of counterfeit versions. Journal of Near Infrared Spectroscopy 16(3) (2008) 327-333.

65. O.Y. Rodionova, A.L. Pomerantsev. NIR-based approach to counterfeit-drug detection. TrAC Trends in Analytical Chemistry 29(8) (2010) 795-803.

66. I. Storme-Paris, H. Rebiere, M. Matoga, C. Civade, P.-A. Bonnet, M.H. Tissier, P. Chaminade. Challenging near infrared spectroscopy discriminating ability for counterfeit pharmaceuticals detection. Analytica Chimica Acta 658(2) (2010) 163-174.

67. M. Blanco, J.Coello, H. Iturriaga, S. Maspoch, C. Pérez-Maseda. Determination of polymorphic purity by near infrared spectrometry. Analytica Chimica Acta 407(12) (2000) 247-254.

68. K.L. Vora, G. Buckton, D. Clapham. The use of dynamic vapour sorption and near infra-red spectroscopy (DVS-NIR) to study the crystal transitions of theophylline and the report of a new solid-state transition. European Journal of Pharmaceutical Sciences 22(2-3) (2004) 97-105.

69. S.E. Barnes, T. Thurston, J.A. Coleman, A. Diederich, D. Ertl, J. Rydzak, P. Ng, K. Bakeev, D. Bhanushali. NIR diffuse reflectance for on-scale monitoring of the polymorphic form transformation of pazopanib hydrochloride (GW786034); Model development and method transfer. Analytical Methods 2(12) (2010) 1890-1899.

70. M. Blanco, D. Valdés, M.S. Bayod, F.Fernández-Marí, J.Llorente. Characterization and analysis of polymorphs by near-infrared spectrometry. Analytica Chimica Acta 502(2) (2004) 221-227.

71. G. Buckton, E. Yonemochi, J. Hammond, A. Moffat. The use of near infra-red spectroscopy to detect changes in the form of amorphous and crystalline lactose. International Journal of Pharmaceutics 168(2) (1998) 231-241.

72. N. Chieng, T. Rades, J. Aaltonen. An overview of recent studies on the analysis of pharmaceutical polymorphs. Journal of Pharmaceutical and Biomedical Analysis 55(4) (2011) 618-644. 
73. W.L. Yoon, R.D. Jee, A. Charvill, G. Lee, A.C. Moffat. Application of near-infrared spectroscopy to the determination of the sites of manufacture of proprietary products. Journal of Pharmaceutical and Biomedical Analysis 34(5) (2004) 933-944.

74. Y. Roggo, N. Jent, A. Edmond, P. Chalus, M. Ulmschneider. Characterizing process effects on pharmaceutical solid forms using near-infrared spectroscopy and infrared imaging. European Journal of Pharmaceutics and Biopharmaceutics 61(1-2) (2005) 100-110.

75. L.E. Rodriguez-Saona, F.M. Khambaty, F.S. Fry, E.M. Calvey. Rapid detection and identification of bacterial strains by fourier transform near-infrared spectroscopy. Journal of Agricultural and Food Chemistry 49(2) (2001) 574-579.

76. L.E. Rodriguez-Saona, F.M. Khambaty, F.S. Fry, J. Dubois, E.M. Calvey. Detection and identification of bacteria in a juice matrix with Fourier transform-near infrared spectroscopy and multivariiate analysis. Journal of Food Protection 67(11) (2004) 2555-2559.

77. M. Otsuka, I. Yamane. Prediction of tablet properties based on near infrared spectra of raw mixed powders by chemometrics: Scale-up factor of blending and tableting processes. Journal of Pharmaceutical Sciences 98(11) (2009) 4296-4305.

78. J. Muselík, K. Krejčová, M. Rabišková, A. Bartošíková, M. Dračková, L. Vorlová. Pellet coating thickness determination by near-infrared reflectance spectroscopy: Comparison of two reference methods. Current Pharmaceutical Analysis 6(4) (2010) 225-233.

79. J. Muselík, K. Krejčová, M. Rabišková, A. Bartošíková, M. Dračková, L. Vorlová. Determination of the thickness of tablet coating by near-infrared spectroscopy. Stanovení tloušt'ky obalu tablet blízkou infračervenou spektroskopií. Chemické listy 104(1) (2010) 41-45.

80. M.C. Sarraguça, A.V. Cruz, H.R. Amaral, P.C. Costa, J.A. Lopes. Comparison of different chemometric and analytical methods for the prediction of particle size distribution in pharmaceutical powders. Analytical and Bioanalytical Chemistry 399(6) (2011) 2137-2147.

81. M.C. Sarraguça, A.V. Cruz, S.O. Soares, H.R. Amaral, P.C. Costa, J.A. Lopes. Determination of flow properties of pharmaceutical powders by near infrared spectroscopy. Journal of Pharmaceutical and Biomedical Analysis 52(4) (2010) 484-492.

82. M.P. Freitas, A. Sabadin, L.M. Silva, F.M. Giannotti, D.A. do Couto, E. Tonhi, R.S. Medeiros, G.L. Coco, V.F.T. Russo, J.A. Martins. Prediction of drug dissolution profiles from tablets using NIR diffuse reflectance spectroscopy: a rapid and nondestructive method. Journal of Pharmaceutical and Biomedical Analysis 39(1-2) (2005) 17-21.

83. H. Mou, X. Wang, T. Lv, L. Xie, H. Xie. On-line dissolution determination of Baicalin in solid dispersion based on near infrared spectroscopy and circulation dissolution system. Chemometrics and Intelligent Laboratory Systems 105(1) (2011) 38-42.

84. K.M. Morisseau, C.T. Rhodes. Near-infrared spectroscopy as a nondestructive alternative to conventional tablet hardness testing. Pharmaceutical Research 14(1) (1997) 108-111.

85. Y. Chen, S.S. Thosar, R.A. Forbess, M.S. Kemper, R.L. Rubinovitz, A.J. Shukla. Prediction of drug content and hardness of intact tablets using artificial neural network and near-infrared spectroscopy. Drug Development and Industrial Pharmacy 27(7) (2001) 623-631. 
86. J.-H. Guo, G.W. Skinner, W.W. Harcum, J.P. Malone, L.G. Weyer. Application of nearinfrared spectroscopy in the pharmaceutical solid dosage form. Drug Development and Industrial Pharmacy 25(12) (1999) 1267-1270.

87. M. Blanco, M. Alcala. Content uniformity and tablet hardness testing of intact pharmaceutical tablets by near infrared spectroscopy: A contribution to process analytical technologies. Analytica Chimica Acta 557(1-2) (2006) 353-359.

88. M. Donoso, E.S. Ghaly. Prediction of drug dissolution from tablets using nearinfrared diffuse reflectance spectroscopy as a nondestructive method. Pharmaceutical Development and Technology 9(3) (2004) 247-265.

89. O. Berntsson, L.G. Danielsson, S. Folestad. Estimation of effective sample size when analyzing powders with diffuse reflectance near-infrared spectrometry. Anaytica Chimica Acta 364(1-3) (1998) 243-251.

90. O. Berntsson, T. Burger, S. Folestad, L.-G. Danielsson, J. Kuhn, J. Fricke. Effective sample size in diffuse reflectance near-IR spectrometry. Analytical Chemistry 71(3) (1999) 617-623.

91. M. Otsuka. Comparative particle size determination of phenacetin bulk powder by using Kubelka-Munk theory and principal component regression analysis based on near-infrared spectroscopy. Powder Technology 141(3) (2004) 244-250.

92. I. Fix, K.-J. Steffens. Quantifying low amorphous or crystalline amounts of alphalactose-monohydrate using X-ray powder diffraction, near-infrared spectroscopy and differential scanning calorimetry. Drug Development and Industrial Pharmacy 30(5) (2004) 513-523.

93. A. Gombas, I. Antal, P. Szabó-Révész, S. Marton, I. Erõs. Quantitative determination of crystallinity of alpha-lactose monohydrate by near infrared spectroscopy (NIRS). International Journal of Pharmaceutics 256(1-2) (2003) 25-32.

94. W. Li, G.D. Worosila, W. Wang, T. Mascaro. Determination of polymorph conversion of an active pharmaceutical ingredient in wet granulation using NIR calibration models generated from the premix blends. Journal of Pharmaceutical Sciences 94(12) (2005) 2800-2806.

95. S.J. Bai, M. Rani, R. Suryanarayanan, J.F. Carpenter, R. Nayar, M.C. Manning. Quantification of glycine crystallinity by near-infrared (NIR) spectroscopy. Journal of Pharmaceutical Sciences 93(10) (2004) 2439-2447.

96. J.J. Seyer, P.E. Luner. Determination of indomethacin crystallinity in the presence of excipients using diffuse reflectance near-infrared spectroscopy. Pharmaceutical Development and Technology 6(4) (2001) 573-582.

97. O. Berntsson, L.-G. Danielsson, M.O. Johnansson and S. Folestad. Quantitative determination of content in binary powder mixtures using diffuse reflectance near infrared spectrometry and multivariate analysis. Analytica Chimica Acta 419(1) (2000) 45-54.

98. G. Fevotte, J. Calas, F. Puel, C. Hoff. Applications of NIR spectroscopy to monitoring and analyzing the solid state during industrial crystallization processes. International Journal of Pharmaceutics 273(1-2) (2004) 159-169.

99. A.D. Patel, P.E. Luner, M.S. Kemper. Quantitative analysis of polymorphs in binary and multi-component powder mixtures by near-infrared reflectance spectroscopy. International Journal of Pharmaceutics 206(1-2) (2000) 63-74. 
100. W.P. Findlay, G.R. Peck, K.R. Morris. Determination of fluidized bed granulation end point using near-infrared spectroscopy and phenomenological analysis. Journal of Pharmaceutical Sciences 94(3) (2005) 604-612.

101. X.P. Zhou, P. Hines, M.W. Borer. Moisture determination in hygroscopic drug substances by near infrared spectroscopy. Journal of Pharmaceutical and Biomedical Analysis 17(2) (1998) 219-225.

102. J. Rantanen, O. Antikainen, J.-P. Mannermaa, J. Yliruusi. Use of the near-infrared reflectance method for measurement of moisture content during granulation. Pharmaceutical Development and Technology 5(2) (2000) 209-217.

103. R.G.Jr. Buice, T.B. Gold, R. A. Lodder, G. A. Digenis. Determination of moisture in intact gelatin capsules by near-infrared spectrophotometry. Pharmaceutical Research 12(1) (1995) 161-163.

104. O. Berntsson, G. Zackrisson, G. Ostling. Determination of moisture in hard gelatin capsules using near-infrared spectroscopy: applications to at-line process control of pharmaceutics. Journal of Pharmaceutical and Biomedical Analysis 15(7) (1997) 895-900.

105. C.C. Corredor, D. Bu, D. Both. Comparison of near infrared and microwave resonance sensors for at-line moisture determination in powders and tablets. Analytica Chimica Acta 696(1-2) (2011) 84-93.

106. N.W. Broad, R.D. Jee, A.C. Moffat, M.J. Eaves, W.C. Mann, W. Dziki. Non-invasive determination of ethanol, propylene glycol and water in a multi-component pharmaceutical oral liquid by direct measurement through amber plastic bottles using Fourier transform near-infrared spectroscopy. Analyst 125(11) (2000) 2054-2058.

107. M. Alcalà, J.Leon, J. Ropero, M. Blanco, R.J. Romañach. Analysis of low content drug tablets by transmission near infrared spectroscopy: Selection of calibration ranges according to multivariate detection and quantitation limits of PLS models. Journal of Pharmaceutical Sciences 97(12) (2008) 5318-5327.

108. J. Ali, K. Pramod, S.H. Ansari. Near-infrared spectroscopy for nondestructive evaluation of tablets. Systematic Reviews in Pharmacy 1(1) (2010) 17-23.

109. R. López-Arellano, E.A. Santander-García, J.M. Andrade-Garda, G. Alvarez-Avila, J.A. Garduño-Rosas, E.A. Morales-Hipólito. Quantification of lysine clonixinate in intravenous injections by NIR spectroscopy. Vibrational Spectroscopy 51(2) (2009) 255-262.

110. P. Singh, D.K. Jangir, R. Mehrotra, A.K. Bakhshi. Development and validation of an infrared spectroscopy-based method for the analysis of moisture content in 5fluorouracil. Drug Testing and Analysis 1(6) (2009) 275-278.

111. M.C. Sarraguça, J.A. Lopes. Quality control of pharmaceuticals with NIR: From lab to process line. Vibrational Spectroscopy 49(2) (2009) 204-210.

112. A.W. Sobanska. Rapid determination of nifuroxazide in tablets by near-infrared spectroscopy. Chemia Analityczna 54(5) (2009) 1021-1033.

113. X. Wang, Q. Fu, J. Sheng, X. Yang, J. Jia, W. Du. Construction of a universal quantitative model for ibuprofen sustained-release capsules from different manufacturers using near-infrared diffuse reflection spectroscopy. Vibrational Spectroscopy 53(2) (2010) 214-217.

114. H. Yang, J. Irudayaraj. Rapid determination of vitamin C by NIR, MIR and FT-Raman techniques. The Journal of Pharmacy and Pharmacology 54(9) (2002) 1247-1255. 
115. P. Chalus, Y. Roggo, S. Freitag, M. Ulmschneider. Comparaison de spéctromètres proche infrarouge pour la détermination de principe actif dans des comprimés à faible dosage. Spectra Analyse 247 (2005) 44-49.

116. P. Chalus, Y. Roggo, S. Walter, M. Ulmschneider. Near-infrared determination of active substance content in intact low-dosage tablets. Talanta 66(5) (2005) 12941302.

117. M. Blanco, J. Coello, H. Iturriaga, S. Maspoch, D. Serrano. Near-infrared analytical control of pharmaceuticals. A single calibration model from mixed phase to coated tablets. Analyst 123(11) (1998) 2307-2312.

118. S.M. Han, P.G. Faulkner. Determination of SB 216469-S during tablet production using near-infrared reflectance spectroscopy. Journal of Pharmaceutical and Biomedical Analysis 14(12) (1996) 1681-1689.

119. W. Li, L. Bagnol, M. Berman, R.A. Chiarella, M. Gerber. Applications of NIR in early stage formulation development. Part II. Content uniformity evaluation of low dose tablets by principal component analysis. International Journal of Pharmaceutics 380(1-2) (2009) 49-54.

120. T. Herkert, H. Prinz, K.-A. Kovar. One hundred percent online identity check of pharmaceutical products by near-infrared spectroscopy on the packaging line. European Journal of Pharmaceutics and Biopharmaceutics 51(1) (2001) 9-16.

121. M. Andersson, S. Folestad, J. Gottfries, M.O. Johansson, M Josefson, K.G. Wahlund. Quantitative analysis of film coating in a fluidized bed process by in-line NIR spectrometry and multivariate batch calibration. Analytical Chemistry 72(9) (2000) 2099-2108.

122. M. Andersson, M. Josefson, F.W. Langkilde, K.-G. Wahlund. Monitoring of a film coating process for tablets using near infrared reflectance spectrometry. Journal of Pharmaceutical and Biomedical Analysis 20(1-2) (1999) 27-37.

123. J.L. Ramirez, M.K. Bellamy, R.J. Romanach. A novel method for analyzing thick tablets by near infrared spectroscopy. AAPS PharmSciTech (2001) 2:E11.

124. I.H.I. Habib, M.S. Kamel. Near infra-red reflectance spectroscopic determination of metformin in tablets. Talanta 60(1) (2003) 185-190.

125. M. Laasonen, T. Harmia-Pulkkinen, C. Simard, M. Rasanen, H. Vuorela. Development and validation of a near-infrared method for the quantitation of caffeine in intact single tablets. Analytical Chemistry 75(4) (2003)754-760.

126. N.W. Broad, R.D. Jee, A.C. Moffat, M.R. Smith. Application of transmission nearinfrared spectroscopy to uniformity of content testing of intact steroid tablets. Analyst 126(12) (2001) 2207-2211.

127. M Blanco, J Coello, H Iturriaga, S Maspoch, N Pou. Development and validation of a near infrared method for the analytical control of a pharmaceutical preparation in three steps of the manufacturing process. Fresenius' Journal of Analytical Chemistry 368(5) (2000) 534-539.

128. A. Eustaquio, M.Blanco, R.D. Jee,and A.C. Moffat. Determination of paracetamol in intact tablets by use of near infrared transmittance spectroscopy. Analytica Chimica Acta 383(3) (1999) 283-290.

129. A.D. Trafford, R.D. Jee, A.C. Moffat, P. Graham. A rapid quantitative assay of intact paracetamol tablets by reflectance near-infrared spectroscopy. Analyst 124 (5) (1999) 163-167. 
130. P. Merckle, K.A. Kovar. Assay of effervescent tablets by near-infrared spectroscopy in transmittance and reflectance mode: acetylsalicylic acid in mono and combination formulations. Journal of Pharmaceutical and Biomedical Analysis 17(3) (1998) 365-374.

131. Y. Dou, Y. Sun, Y.Ren, P.Ju, Y. Ren. Simultaneous non-destructive determination of two components of combined paracetamol and amantadine hydrochloride in tablets and powder by NIR spectroscopy and artificial neural networks. Journal of Pharmaceutical and Biomedical Analysis 37(3) (2005) 543-549.

132. P. Corti, E. Dreassi, G. Ceramelli, S. Mattii. Near infrared transmittance analysis for the assay of solid pharmaceutical dosage forms. Analyst 124(5) (1999) 755-758.

133. Y. Dou, Y. Sun, Y. Ren, Y. Ren. Artificial neural network for simultaneous determination of two components of compound paracetamol and diphenhydramine hydrochloride powder on NIR spectroscopy. Analytica Chimica Acta 528(1) (2005) 55-61.

134. C.C. Fertig, F. Podczeck, R.D. Jee, M.R. Smith. Feasibility study for the rapid determination of the amylose content in starch by near-infrared spectroscopy. European Journal of Pharmaceutical Sciences 21(2-3) (2004) 155-159.

135. M. Blanco, J. Coello, A. Eustanquio, H. Iturriaga, S. Maspock. Development and validation of a method for the analysis of a pharmaceutical preparation by nearinfrared diffuse reflectance spectroscopy. Journal of Pharmaceutical Sciences 88(5) (1999) 551-556.

136. G.X. Zhou, Z. Ge, J. Dorwart, B. Izzo, J. Kukura, G. Bickel, J. Wyvratt. Determination and differentiation of surface and bound water in drug substances by near infrared spectroscopy. Journal of Pharmaceutical Sciences 92(5) (2003) 1058-1065.

137. S.V.S. Tumuluri, S. Prodduturi, M.M. Crowley, S.P. Stodghill, J.W. McGinity, M.A. Repka, B.A. Avery. The use of near-infrared spectroscopy for the quantitation of a drug in hot-melt extruded films. Drug Development and Industrial Pharmacy 30(5) (2004) 505-511.

138. M.S. Kemper, E.J. Magnuson, S.R. Lowry, W.J. McCarthy, N. Aksornkoae, D.C. Watts, J.R. Johnson, A.J. Shukla. Use of FT-NIR transmission spectroscopy for the quantitative analysis of an active ingredient in a translucent pharmaceutical topical gel formulation. AAPS PharmSci. 3(3) (2001) E23.

139. D.J. Wargo, J.K. Drennen. Near-infrared spectroscopic characterization of pharmaceutical powder blends. Journal of Pharmaceutical and Biomedical Analysis 14(11) (1996) 1415-1423.

140. R.P. Cogdill, C.A. Anderson, M. Delgado-Lopez, D. Molseed, R. Chisholm, R. Bolton, T. Herkert, A.M. Afnán, J.K.3rd Drennen. Process analytical technology case study part I: feasibility studies for quantitative near-infrared method development. AAPS PharmSciTech 6(2) (2005) E262-E272.

141. R.P. Cogdill, C.A. Anderson, M. Delgado-Lopez, D. Molseed, R. Chisholm, R. Bolton, T. Herkert, A.M. Afnán, J.K.3rd Drennen. Process analytical technology case study: part II. Development and validation of quantitative near-infrared calibrations in support of a process analytical technology application for real-time release. AAPS PharmSciTech 6(2) (2005) E273-E283.

142. R.P. Cogdill, C.A. Anderson, J.K. Drennen. Process Analytical Technology Case Study, Part III: Calibration Monitoring and Transfer. AAPS PharmSciTech. 6(2) (2005) E284-E297. 
143. J. Cho, P.J. Gemperline, P.K. Aldridge, S.S. Sekulic. Effective mass sampled by NIR fiber-optic reflectance probes in blending processes. Analytica Chimica Acta 348(1-3) (1997) 303-310.

144. P.A Hailey, P. Doherty, P. Tapsell, T. Oliver, P.K. Aldridge. Automated system for the on-line monitoring of powder blending processes using near-infrared spectroscopy part I. System development and control. Journal of Pharmaceutical and Biomedical Analysis 14(5) (1996) 551-559.

145. S.S. Sekulic, H.W. Ward, D.R. Brannegan, E.D. Stanley, C.L. Evans, S.T. Sciavolino, P.A. Hailey, P.K. Aldridge. On-line monitoring of powder blend homogeneity by nearinfrared spectroscopy. Analytical Chemistry 68(3) (1996) 509-513.

146. S.S. Sekulic, J. Wakenman, P. Doherty, P.A. Hailey. Automated system for the on-line monitoring of powder blending processes using near-infrared spectroscopy. Part II. Qualitative approaches to blend evaluation. Journal of Pharmaceutical and Biomedical Analysis 17(8) (1998) 1285-1309.

147. E.T.S. Skibsted, H.F.M. Boelens, J.A. Westerhuis, D.T. Witte, A.K. Smilde. Simple assessment of homogeneity in pharmaceutical mixing processes using a nearinfrared reflectance probe and control charts. Journal of Pharmaceutical and Biomedical Analysis 41(1) (2006) 26-35.

148. M. Popo, S. Romero-Torres, C. Conde, R.J. Romañach. Blend uniformity analysis using stream sampling and near infrared spectroscopy. AAPS PharmSciTech 3(3) (2002) E24.

149. O. Berntsson, L.-G. Danielsson, B. Lagerholm, S. Folestad. Quantitative in-line monitoring of powder blending by near infrared reflection spectroscopy. Powder Technology 123(2-3) (2002) 185-193.

150. Y. Ghebremeskel, R.A. Weiss. Near infrared analysis of blends of sulfonated polystyrene ionomers and poly(E-caprolactam). Journal of Polymer Science, Part B: Polymer Physics 46(15) (2008) 1602-1610.

151. H. Ma, C.A. Anderson. Characterization of pharmaceutical powder blends by NIR chemical imaging. Journal of Pharmaceutical Sciences 97(8) (2008) 3305-3320.

152. Z. Shi, R.P. Cogdill, S.M. Short, C.A. Anderson. Process characterization of powder blending by near-infrared spectroscopy: Blend end-points and beyond. Journal of Pharmaceutical and Biomedical Analysis 47(4-5) (2008) 738-745.

153. I. Storme-Paris, J. Clarot, S. Esposito, J.C. Chaumeil, A. Nicolas, F. Brion, A. Rientord, P. Chaminade. Near infrared Spectroscopy homogeneity evaluation of complex powder blends in a small-scale pharmaceutical preformulation process, a real-life application. European Journal of Pharmaceutics and Biopharmaceutics 72(1) (2001) 189-198.

154. C.C. Corredor, D. Jayawickrama, G. McGeorge, D.B.B.M. Squibb, Monitoring of blending uniformity: Form conversion and fluid bed drying by near infrared and Raman spectroscopy. American Pharmaceutical Review 13(1) (2010) 66-72 January/February2010.

155. S.Kim, H. Nakagawa, M. Kano, S. Hasebe. Estimation of active pharmaceutical ingredients content in blending process for drug products manufacturing. AIChE Annual Meeting, paper 445d, Salt Lake City, USA, Nov. 7-12 (2010).

156. C.V. Liew, A.D. Karande, P.W.S. Heng. In-line quantification of drug and excipients in cohesive powder blends by near infrared spectroscopy. International Journal of Pharmaceutics 386(1-2) (2010) 138-148. 
157. T. Puchert, C.-V. Holzhauer, J.C. Menezes, D. Lochmann, G. Reich. A new PAT/QbD approach for the determination of blend homogeneity: Combination of on-line NIRS analysis with PC Scores Distance Analysis (PC-SDA). European Journal of Pharmaceutics and Biopharmaceutics 78(1) (2011) 173-182.

158. P. Doležel, J. Muselík, K. Dvořáčková, K. Šustová. A study of the process of homogenization of powder mixtures using NIR spectroscopy. Studium procesu homogenizace práškových směsí s využitím NIR spektroskopie. Česká a slovenská farmacie 59(6) (2010) 263-271.

159. D.M. Koller, A. Posch, G. Hörl, C. Voura, S. Radl, N. Urbanetz, S.D. Fraser, W. Tritthart, F. Reiter, M. Schlingmann, J.G. Khinast. Continuous quantitative monitoring of powder mixing dynamics by near-infrared spectroscopy. Powder Technology 205(1-3) (2011) 87-96.

160. A.U. Vanarase, M. Alcalà, J.I. Jerez Rozo, F.J. Muzzio, R.J. Romañach. Real-time monitoring of drug concentration in a continuous powder mixing process using NIR spectroscopy. Chemical Engineering Science 65(21) (2010) 5728-5733.

161. J. Rantanen, S. Lehtola, P. Rämet, J.-P. Mannermaa, J. Yliruusi. On-line monitoring of moisture content in an instrumented fluidized bed granulator with a multi-channel NIR moisture sensor. Powder Technology 99(2) (1998) 163-170.

162. J. Rantanen, E. Räsänen, J. Tenhunen, M. Känsäkoski, J.-P. Mannermaa, J. Yliruusi. In-line moisture measurement during granulation with a four-wavelength near infrared sensor: an evaluation of particle size and binder effects. European Journal of Pharmaceutics and Biopharmaceutics 50(2) (2000) 271-276.

163. J. Rantanen, E. Rasanen, O. Antikainen, J.-P. Mannermaa, J. Yliruusi. In-line moisture measurement during granulation with a four-wavelength near-infrared sensor: an evaluation of process-related variables and a development of non-linear calibration model. Chemometrics and Intelligent Laboratory Systems 56(1) (2001) 51-58.

164. P. Frake, D. Greenhalgh, S.M. Grierson, J.M. Hempenstall, D.R. Rudd. Process control and end-point determination of a fluid bed granulation by application of near infrared spectroscopy. International Journal of Pharmaceutics 151(1) (1997) 75-80.

165. A. Gupta, G.E. Peck, R.W. Miller, K.R. Morris. Real-time near-infrared monitoring of content uniformity, moisture content, compact density, tensile strength, and young's modulus of roller compacted powder blends. Journal of Pharmaceutical Sciences 94(7) (2005)1589-1597.

166. M. Alcalà, M. Blanco, M. Bautista, J.M. González. On-line monitoring of a granulation process by NIR spectroscopy. Journal of Pharmaceutical Sciences 99(1) (2010) 336-345.

167. A.T. Tok, X. Goh, W.T. Ng, R.B. Tan. Monitoring granulation rate processes using three PAT tools in a pilot-scale fluidized bed. AAPS PharmSciTech 9(4) (2008) 1083-1091.

168. M. Brülls, S. Folestad, A. Sparén, A. Rasmuson. In-Situ Near-Infrared Spectroscopy Monitoring of the Lyophilization Process. Pharmaceutical research 20(3) (2003) 494-499.

169. L. Sukowski, M. Ulmschneider. In-line process analytical technology based on qualitative near-infrared spectroscopy modeling. Pharmazeutische Industrie 67(7) (2005) 830-835. 
170. A. Peinado, J. Hammond, A. Scott. Development, validation and transfer of a near infrared method to determine in-line the end point of a fluidised drying process for commercial production batches of an approved oral solid dose pharmaceutical product. Journal of Pharmaceutical and Biomedical Analysis 54(1) (2011) 13-20.

171. T.D. Davis, G.E. Peck, J.G. Stowell, K.R. Morris, S.R. Byrn. Modeling and Monitoring of Polymorphic Transformations During the Drying Phase of Wet Granulation. Pharmaceutical Research 21(5) (2004) 860-866.

172. J.D. Pérez-Ramos, W. P. Findlay, G. Peck, K.R. Morris. Quantitative analysis of film coating in a pan coater based on in-line sensor measurements. AAPS PharmSciTech, 6(1) (2005) E127-E136.

173. A. Bogomolov, M. Engler, M. Melichar, A. Wigmore. In-line analysis of a fluid bed pellet coating process using a combination of near infrared and Raman spectroscopy. Journal of Chemometrics 24(7-8) (2010) 544-557.

174. M. Bürmen, M. Možna, F. Pernuš, B. Likar. Coating film thickness of pharmaceutical pellets measured by near infrared spectroscopy. Industrial Technology (ICIT), 2010 IEEE International Conference (2010) 199-204.

175. M.-J. Lee, D.-Y. Seo, H.-E. Lee, I.-C. Wang, W.-S. Kim, M.-Y. Jeong, G.J. Choi. In line NIR quantification of film thickness on pharmaceutical pellets during a fluid bed coating process. International Journal of Pharmaceutics 403(1-2) (2011) 66-72.

176. M.J. Lee, C.R. Park, A.Y. Kim, B.S. Kwon, K.H. Bang, Y.S. Cho, M.Y. Jeong, G.J. Choi. Dynamic calibration for the in-line NIR monitoring of film thickness of pharmaceutical tablets processed in a fluid-bed coater. Journal of Pharmaceutical Sciences 99(1) (2010) 325-335.

177. Arnold, et al., Employing Near-Infrared Spectroscopic Methods of Analysis for Fermentaion Monitoring and Control Part 1, Method Development. BioPharm International, 2002. November 2002. 26-34.

178. Arnold, et al., Employing Near-Infrared Spectroscopic Methods of Analysis for Fermentaion Monitoring and Control Part 2, Implementation Strategies. BioPharm International, 2003. January 2003. 47-70.

179. C. Cimander, C.-F. Mandenius. Online monitoring of a bioprocess based on a multianalyser system and multivariate statistical process modelling. Journal of Chemical Technology \& Biotechnology 77(10) (2002) 1157-1168.

180. M. Navrátil, A. Norberg, L. Lembrén, C.-F. Mandenius. On-line multi-analyzer monitoring of biomass, glucose and acetate for growth rate control of a vibrio cholerae fed-batch cultivation. Journal of Biotechnology 115(1) (2005) 67-79.

181. E. Tamburini, G. Vaccari, S. Tosi, A. Trilli. Near-Infrared Spectroscopy: A Tool for Monitoring Submerged Fermentation Processes Using an Immersion Optical-Fiber Probe. Applied Spectroscopy 57(2) (2003) 132-138.

182. S. Tosi, M. Rossi, E. Tamburini, G. Vaccari, A. Amaretti, D. Matteuzzi. Assessment of in-line near-infrared spectroscopy for continuous monitoring of fermentation processes. Biotechnology Progress 19(6) (2003) 1816-1821.

183. K.S.Y. Yeung, M. Hoare, N. F. Thornhill, T. Williams, J.D. Vaghjiani. Near-Infrared Spectroscopy for Bioprocess Monitoring and Control. Biotechnology and Bioengineering 63(6) (1998) 684-693.

184. S. Alison Arnold, J. Crowley, N. Woods, L.M. Harvey, B. McNeil. In-situ near infrared spectroscopy to monitor key analytes in mammalian cell cultivation. Biotechnology and Bioengineering 84(1) (2003) 13-19. 
185. M.R. Riley, M. Rhiel, X. Zhou, M.A. Arnold, D.W. Murhammer. Simultaneous measurement of glucose and glutamine in insect cell culture media by near infrared spectroscopy. Biotechnology and Bioengineering 55(1) (1996) 11-15.

186. C.B. Lewis, R.J. McNichols, A. Gowda, G.L. Coté. Investigation of Near-Infrared Spectroscopy for Periodic Determination of Glucose in Cell Culture Media in Situ. Applied Spectroscopy 54(10) (2000) 1453-1457.

187. B. Jung, S.Lee, J.H. Yang, T. Good, G.L. Coté. Automated On-Line Noninvasive Optical Glucose Monitoring in a Cell Culture System. Applied Spectroscopy 56(1) (2002) 51-57.

188. D. Landgrebe, C. Haake, T. Höpfner, S. Beutel, B. Hitzmann, T. Scheper, M. Rhiel, K. Reardon. On-line infrared spectroscopy for bioprocess monitoring. Applied Microbiology and Biotechnology 88(1) (2010) 11-22.

189. A.S. Rathore, Bhambure, V. Ghare. Process analytical technology (PAT) for biopharmaceutical products. Analytical and Bioanalytical Chemistry 398(1) (2010) 137-154.

190. E.K. Read, R.B. Shah, B.S. Riley, J.T. Park, K.A. Brorson, A.S. Rathore. Process analytical technology (PAT) for biopharmaceutical products: Part II. Concepts and applications. Biotechnology and Bioengineering 105(2) (2010) 285-295.

191. P. Roychoudhury, R.O`Kennedy, B. McNeil, L.H. Harvey. Multiplexing fibre optic near infrared (NIR) spectroscopy as an emerging technology to monitor industrial bioprocesses. Analytica Chimica Acta 590(1) (2007) 110-117.

192. Z.I.T.A. Soons, M. Streefland, G. van Straten, A.J.B. van Boxtel. Assessment of near infrared and "software sensor" for biomass monitoring and control. Chemometrics and Intelligent Laboratory Systems 94(2) (2008) 166-174.

193. A.P. Teixeira, R. Oliveira, P.M. Alves, M.J. T. Carrondo. Advances in on-line monitoring and control of mammalian cell cultures: Supporting the PAT initiative. Biotechnology Advances 27(6) (2009) 726-732.

194. J.G. Henriques, S. Buziol, E. Stocker, A. Voodg, J.C. Menezer. Monitoring mammalian cell cultivations for monoclonal antibody production using near-infrared spectroscopy. Advances in Biochemical Engineering / Biotechnology 116 (2010) 73-97.

195. Y. Roggo, C. Roeseler, M. Ulmschneider. Near infrared spectroscopy for qualitative comparison of pharmaceutical batches. Journal of Pharmaceutical and Biomedical Analysis 36(4) (2004) 777-786.

196. Y. Roggo, L. Duponchel, J.-P. Huvenne. Comparison of supervised pattern recognition methods with McNemar's statistical test: Application to qualitative analysis of sugar beet by near-infrared spectroscopy. Analytica Chimica Acta 477(2) (2003) 187-200.

197. C. Beleites, G. Steiner, M.G. Sowa, R. Baumgartner, S. Sobottka, G. Schackert, R. Salzer. Classification of human gliomas by infrared imaging spectroscopy and chemometric image processing. Vibrational Spectroscopy 38(1-2) (2005) 143-149.

198. J.M. Chalmers, N.J. Everall, M.D. Schaeberle, I.W. Levin, E.Neil Lewis, L.H. Kidder, J. Wilson, R. Crocombe. FT-IR imaging of polymers: an industrial appraisal. Vibrational Spectroscopy 30(1) (2002) 43-52.

199. A.d. Juan, R. Tauler, R. Dyson, C. Marcolli, M. Rault, M. Maeder. Spectroscopic imaging and chemometrics: a powerful combination for global and local sample analysis. TrAC Trends in Analytical Chemistry 23(1) (2004) 70-79.

200. E.N. Lewis, J. Schoppelrei, E. Lee. Near-infrared chemical Imaging and the PAT initiative. Spectroscopy 19(4) (2004) 26-36. 
201. E.N. Lewis, E. Lee, L.H. Kidder. Combining Imaging and Spectroscopy: Solving Problems with Near Infrared Chemical Imaging. Microscopy Today 12(6) (2004) 8-12.

202. C. Gendrin, Y. Roggo, C. Collet. Pharmaceutical applications of vibrational chemical imaging and chemometrics: A review. Journal of Pharmaceutical and Biomedical Analysis 48(3) (2008) 533-553.

203. R.A. Lodder. NIR spectrometric imaging. European Journal of Pharmaceutical Sciences 2(1-2) (1994) 84.

204. M. Blanco, R. Cueva-Mestanza, A. Peguero. Controlling individual steps in the production process of paracetamol tablets by use of NIR spectroscopy. Journal of Pharmaceutical and Biomedical Analysis 51(4) (2010) 797-804.

205. M. Blanco, M. Bautista, M. Alcalà. API determination by NIR spectroscopy across pharmaceutical production process. AAPS PharmSciTech 9(4) (2008) 1130-1135

206. Y. Roggo, K. Dégardin, P. Margot. Identification of pharmaceutical tablets by Raman spectroscopy and chemometrics. Talanta 81(3) (2010) 988-995.

207. Y. Roggo, A. Edmond, P. Chalus, M. Ulmschneider. Infrared hyperspectral imaging for qualitative analysis of pharmaceutical solid forms. Analytica Chimica Acta 535(1-2) (2005) 79-87. 